\title{
Quantum computational logic with mixed states
}

\author{
H. Freytes* ${ }^{1,2}$ and G. Domenech*3 \\ 1. Instituto Argentino de Matemática (IAM) \\ Saavedra 15 - 3er piso - 1083 Buenos Aires, Argentina \\ 2. Universita degli Studi di Cagliari \\ Via Is Mirrionis 1, 09123, Cagliari - Italia \\ 3. Instituto de Astronomía y Física del Espacio (IAFE) \\ Casilla de Correo 67, Sucursal 28, 1428 Buenos Aires - Argentina
}

\begin{abstract}
In this paper we solve a problem posed in [3] and [5] about the axiomatizability of a system of quantum computational gates known as the Poincaré irreversible quantum computational system. A Hilbertstyle calculus is introduced obtaining a strong completeness theorem.

Keywords: Quantum computational logic, $M V$-algebras, $P M V$-algebras. Mathematics Subject Classification 2000: 06B99, 03B52, 06D35.
\end{abstract}

\section{Introduction}

The idea of quantum computation was introduced in 1982 by Richard Feynmann and remained primarily of theoretical interest until developments such as the invention of an algorithm to factor large numbers triggered a vast domain of research. In a classical computer, information is encoded in a series of bits and these bits are manipulated via Boolean logical gates like NOT, $O R, A N D$, etc, arranged in succession to produce an end result. Standard quantum computing is based on quantum systems described by finite dimensional Hilbert spaces, specially $\mathbb{C}^{2}$-the two-dimensional space of a qbit. A qbit (the quantum counterpart of the classical bit) is represented by a

${ }^{*}$ Fellow of the Consejo Nacional de Investigaciones Científicas y Técnicas (CONICET) 
unit vector in $\mathbb{C}^{2}$ and, generalizing for a positive integer $n, n$-qbits are represented by unit vectors in $\mathbb{C}^{2^{n}}$. Similarly to the classical computing case, we can introduce and study the behavior of a number of quantum logical gates (hereafter quantum gates for short) operating on qbits. Quantum computing can simulate all computations which can be done by classical systems; however, one of the main advantages of quantum computation and quantum algorithms is that they can speed up computations [19].

In [3] and [5], a quantum gate system called Poincaré irreversible quantum computational system (IIP-system for short) was developed. The $\mathbb{I P}$ system is an interesting set of quantum gates specially for two reasons: i) it is related to continuous $t$-norms [17], i.e. continuous binary operations on the interval $[0,1]$ that are commutative, associative and non-decreasing with 1 is the unit element. They are naturally proposed as interpretations of the conjunction in systems of fuzzy logic [13]. ii) Subsequent generalizations allow to connect the $\mathbb{I P}$-system with sequential effect algebras [10], introduced to study the sequential action of quantum effects which are unsharp versions of quantum events $[11,12]$.

Our work is motivated by the $\mathbb{I P}$-system, and mainly by the following question proposed by the authors in [3] and [5]: "The axiomatizability of quantum computational logic is an open problem.". To answer this claim, we study an algebraic structure related to the $\mathbb{P} \mathbb{P}$-system and we provide a Hilbert-style calculus, obtaining a strong completeness theorem with respect to the mentioned structure.

The paper is structured as follows: In Section 1, we briefly resume basic physical notions of mathematical approaches to quantum computation. Section 2 contains generalities on universal algebra and algebraic structures associated with Łukasiewicz infinite-valued calculus as $M V$-algebras and product $M V$-algebras. In Section 3 we introduce a set of quantum gates known as Poincaré irreversible quantum gates system. The mathematical representation of these quantum gates is closely related with the product $M V$-algebras structure. In Section 4, algebraic structures associated to quantum computation are introduced. Specifically, we introduce an expansion of the equational class known as square root quasi $M V$-algebras [9], expansion that we call "square root quasi $P M V$-algebra" (or $\sqrt{q P M V}$-algebra for short). In Section 5 we focus on a subvariety of the $\sqrt{q P M V}$-algebras called Irreversible Poincaré Algebras. In Section 6 we introduce the notion of probabilistic consequence. It provides a generalization of a classical problem related to digital circuits which consists in knowing whether a determinate state of the output of a set of a circuits conditions a determine state of 
the output of another circuit. Finally, in Section 7 we give a Hilbert-style axiomatization, called $\mathcal{L} \mathcal{I} \mathcal{P}$, for the probabilistic consequence. A strong completeness theorem for $\mathcal{L I P}$ with respect to the variety of Irreversible Poincaré Algebras is obtained.

\section{Basic notions in quantum computation}

In quantum computation, information is elaborated and processed by means of quantum systems. The pure state of a quantum system is described by a unit vector in a Hilbert space, denoted by $|\varphi\rangle$ in Dirac notation. A quantum bit or qbit, the fundamental concept of quantum computation, is a pure state in the Hilbert space $\mathbb{C}^{2}$. The standard orthonormal basis $\{|0\rangle,|1\rangle\}$ of $\mathbb{C}^{2}$ where $|0\rangle=(1,0)$ and $|1\rangle=(0,1)$ is called the logical basis. Thus, pure states $|\varphi\rangle$ in $\mathbb{C}^{2}$ are coherent superpositions of the basis vectors with complex coefficients

$$
|\psi\rangle=c_{0}|0\rangle+c_{1}|1\rangle, \quad \text { with } \quad\left|c_{0}\right|^{2}+\left|c_{1}\right|^{2}=1
$$

Recalling the Born rule, any qubit $|\psi\rangle=c_{0}|0\rangle+c_{1}|1\rangle$ may be regarded as a piece of information, where the number $\left|c_{0}\right|^{2}$ corresponds to the probabilityvalue of the information described by the basic state $|0\rangle$; while $\left|c_{1}\right|^{2}$ corresponds to the probability-value of the information described by the basic state $|1\rangle$. The two basis-elements $|0\rangle$ and $|1\rangle$ are usually taken as encoding the classical bit-values 0 and 1 , respectively. By these means, a probability value is assigned to a qbit as follows:

Definition 1.1 [3], [5] Let $|\psi\rangle=c_{0}|0\rangle+c_{1}|1\rangle$ be a qbit. Then its probability value is $p(|\psi\rangle)=\left|c_{1}\right|^{2}$

The quantum states of interest in quantum computation lie in the tensor product $\otimes^{n} \mathbb{C}^{2}=\mathbb{C}^{2} \otimes \mathbb{C}^{2} \otimes \ldots \otimes \mathbb{C}^{2}(n$ times $)$, a $2^{n}$-dimensional complex space. A special basis, called the $2^{n}$-computational basis, is chosen for $\otimes^{n} \mathbb{C}^{2}$. More precisely, it consists of the $2^{n}$ orthogonal states $|\iota\rangle, 0 \leq \iota \leq 2^{n}$ where $\iota$ is in binary representation and $|\iota\rangle$ can be seen as tensor product of states (Kronecker product) $|\iota\rangle=\left|\iota_{1}\right\rangle \otimes\left|\iota_{2}\right\rangle \otimes \ldots \otimes\left|\iota_{n}\right\rangle$ where $\iota_{j} \in\{0,1\}$. A pure state $|\psi\rangle \in \otimes^{n} \mathbb{C}^{2}$ is a superposition of the basis vectors $|\psi\rangle=\sum_{\iota=1}^{2^{n}} c_{\iota}|\iota\rangle$ with $\sum_{\iota=1}^{2^{n}}\left|c_{\iota}\right|^{2}=1$.

In the usual representation of quantum computational processes, a quantum circuit is identified with an appropriate composition of quantum gates, 
i.e. unitary operators acting on pure states of a convenient ( $n$-fold tensor product) Hilbert space $\otimes^{n} \mathbb{C}^{2}[25]$. Consequently, quantum gates represent time reversible evolutions of pure states of the system.

In general, a quantum system is not in a pure state. This may be caused, for example, by the non complete efficiency in the preparation procedure or by the fact that systems cannot be completely isolated from the environment, undergoing decoherence of their states. On the other hand, there are interesting processes that cannot be encoded in unitary evolutions, for example, at the end of the computation a non-unitary operation, a measurement, is applied, and the state becomes a probability distribution over pure states, or what is called a mixed state. In view of these facts, several authors $[1,10,27]$ have paid attention to a more general model of quantum computational processes, where pure states are replaced by mixed states. This model is known as quantum computation with mixed states. In what follows we briefly describe the mentioned model.

Let $H$ be a complex Hilbert space. We denote by $\mathcal{L}(H)$ the space of linear operators on $H$. In the model of quantum computation with mixed states, we regard a quantum state in a Hilbert space $H$ as a density operator i.e., an Hermitian operator $\rho \in \mathcal{L}(H)$ that is positive semidefinite $(\rho \geq 0)$ and has unit trace $(\operatorname{tr}(\rho)=1)$. We denote by $\mathcal{D}(H)$ the set of all density operators in $H$. A quantum operation is a linear map $\mathcal{E}: \mathcal{L}\left(H_{1}\right) \rightarrow \mathcal{L}\left(H_{2}\right)$ that is tracepreserving and completely positive. Intuitively, completely positive means that if we embed $H$ into some larger system, the standard lifting of $\mathcal{E}$ to the larger system preserves positive definiteness, and thus states get mapped to states. Formally, this means that for any Hilbert space $K$, the linear map $\mathcal{E} \otimes \mathcal{I}_{K}: \mathcal{L}\left(H_{1} \otimes K\right) \rightarrow \mathcal{L}\left(H_{2} \otimes K\right)$ where $\mathcal{I}_{K}$ is the identity in $\mathcal{L}(H)$, satisfies that for any $\rho \in \mathcal{L}\left(H_{1} \otimes K\right)$, if $\rho>0$ then $\left(\mathcal{E} \otimes \mathcal{I}_{K}\right)(\rho)>0$. Each quantum operation $\mathcal{E}$ may be expressed as $\mathcal{E}(\rho)=\sum_{i} A_{i} \rho A_{i}^{\dagger}$ where $A_{i}$ are linear operators satisfying $\sum_{i} A_{i}^{\dagger} A_{i}=I$ (Kraus representation [18]).

In the representation of quantum computational processes based on mixed states, a quantum circuit is a circuit whose inputs and outputs are labeled with density operators and whose quantum gates are labeled with quantum operations. In terms of density operators, a $n$-qbit $|\psi\rangle \in \otimes^{n} \mathbb{C}^{2}$ can be represented as a matrix product $\rho=|\psi\rangle\langle\psi|$, where $\langle\psi|=| \psi\rangle^{\dagger}$. Moreover, every unitary operator $U$ on a Hilbert space $\otimes^{m} \mathbb{C}^{2}$ gives rise to a quantum operation $\mathcal{O}_{\mathcal{U}}$ such that $\mathcal{O}_{\mathcal{U}}(\sigma)=\mathcal{U} \sigma \mathcal{U}^{\dagger}$ for each $\sigma \in \mathcal{L}(H)$. In fact, quantum computation with mixed states is a generalization of the standard model based on qbits and unitary operations. We want to stress that the 
measurement process in quantum computation can also be described by a quantum operation, an important fact that reinforces the election of quantum operations to represent quantum gates. We refer to [1, 25, 27], for more details and motivations about quantum operations.

In this powerful model we can extend, in a natural way, the logical base of qbits and the notion of probability assigned to a qbit. In fact: we may relate to each vector of the logical basis of $\mathbb{C}^{2}$ one of the distinguished density operators $P_{0}=|0\rangle\langle 0|$ and $P_{1}=|1\rangle\langle 1|$ that represent the falsity-property and the truth-property respectively. Generalizing to the framework of $n$ dimensions, the two special operators $P_{0}^{(n)}=\frac{1}{\operatorname{Tr}\left(I^{n-1} \otimes P_{0}\right)} I^{n-1} \otimes P_{0}$ and $P_{1}^{(n)}=\frac{1}{\operatorname{Tr(I^{n-1}\otimes P_{1})}} I^{n-1} \otimes P_{1}$ (where $n$ is even and $n \geq 2$ ) represent, in each space $\mathcal{D}\left(\otimes^{n} \mathbb{C}^{2}\right)$, the falsity-property and the truth-property respectively. By applying the Born rule, the probability to obtain the truth-property $P_{1}^{(n)}$ for a system being in the state $\rho$ is given by the following definition:

Definition 1.2 [3], [5] Let $\rho \in \mathcal{D}\left(\otimes^{n} \mathbb{C}^{2}\right)$. Then, its probability value is $p(\rho)=\operatorname{Tr}\left(P_{1}^{(n)} \rho\right)$.

Note that, in the particular case in which $\rho=|\psi\rangle\langle\psi|$ where $|\psi\rangle=c_{0}|0\rangle+$ $c_{1}|1\rangle$, we obtain that $p(\rho)=\left|c_{1}\right|^{2}$. This definition of probability allows to introduce a binary relation $\leq_{w}$ on $\mathcal{D}\left(\otimes^{n} \mathbb{C}^{2}\right)$ in the following way:

$$
\sigma \leq_{w} \rho \quad \text { iff } \quad p(\sigma) \leq p(\rho)
$$

One can easily see that $\left\langle\mathcal{D}\left(\otimes^{n} \mathbb{C}^{2}\right), \leq_{w}\right\rangle$ is a preorder and it will play an important role in the rest of the paper.

\section{$2 \quad M V$-algebras and $P M V$-algebras}

We freely use all basic notions of universal algebra that can be found in [2]. Let $\sigma$ be a type of algebras and let $\mathcal{A}$ be a class of algebras of type $\sigma$. We denote by $\operatorname{Term}_{\mathcal{A}}$ the absolutely free algebra of type $\sigma$ built from the set of variables $V=\left\{x_{1}, x_{2}, \ldots\right\}$. Each element of $\operatorname{Term}_{\mathcal{A}}$ is referred as an $\mathcal{A}$-term. For $t \in \operatorname{Term}_{\mathcal{A}}$ we often write $t$ as $t\left(x_{1}, x_{2}, \ldots, x_{n}\right)$ to indicate that the variables occurring in $t$ are among $x_{1}, x_{2}, \ldots, x_{n}$. Let $A \in \mathcal{A}$. If $t\left(x_{1}, x_{2}, \ldots, x_{n}\right) \in \operatorname{Term}_{\mathcal{A}}$ and $a_{1}, \ldots a_{n} \in A$, by $t^{A}\left[a_{1}, \ldots, a_{n}\right]$, we denote the result of the application of the term operation $t^{A}$ to the elements $a_{1}, \ldots a_{n} \in A$. A valuation in $A$ is a map $v: V \rightarrow A$. Of course, any valuation $v$ in $A$ can be uniquely extended to an $\mathcal{A}$-homomorphism $v: \operatorname{Term}_{\mathcal{A}} \rightarrow$ 
$A$ in the usual way, i.e., if $t_{1}, \ldots, t_{n} \in \operatorname{Term}_{\mathcal{A}}$ then $v\left(t\left(t_{1}, \ldots, t_{n}\right)\right)=$ $t^{A}\left(v\left(t_{1}\right), \ldots, v\left(t_{n}\right)\right)$. Thus, valuations are identified with $\mathcal{A}$-homomorphisms from the absolutely free algebra. If $t, s \in \operatorname{Term}_{\mathcal{A}}, A=t=s$ means that for each valuation $v$ in $A, v(t)=v(s)$ and $\mathcal{A}=t=s$ means that for each $A \in \mathcal{A}$, $A=t=s$. If $\mathcal{S}$ is a subclass of $\mathcal{A}, \mathcal{V}(\mathcal{S})$ denotes the variety generated by $\mathcal{S}$.

Now we introduce some basic notions in algebraic structures associated to fuzzy logic. An $M V$-algebra [4] is an algebra $\langle A, \oplus, \neg, 0\rangle$ of type $\langle 2,2,0\rangle$ satisfying the following equations:

MV1 $\langle A, \oplus, 0\rangle$ is an abelian monoid,

$\mathrm{MV} 2 \neg \neg x=x$,

MV3 $x \oplus \neg 0=\neg 0$,

MV4 $\neg(\neg x \oplus y) \oplus y=\neg(\neg y \oplus x) \oplus x$.

In agreement with the usual $M V$-algebraic operations we define:

$$
\begin{array}{ll}
x \odot y=\neg(\neg x \oplus \neg y), & x \rightarrow y=\neg x \oplus y, \\
x \wedge y=x \odot(x \rightarrow y), & 1=\neg 0, \\
x \vee y=(x \rightarrow y) \rightarrow y . &
\end{array}
$$

On each $M V$-algebra $A$ we can define an order $x \leq y$ iff $x \rightarrow y=1$. This order turns $\langle A, \wedge, \vee, 0,1\rangle$ into a distributive bounded lattice with 1 the greatest element and 0 the smallest element.

A very important example of $M V$-algebra is $[0,1]_{M V}=\langle[0,1], \oplus, \neg, 0\rangle$ such that $[0,1]$ is the real unit segment and $\oplus$ and $\neg$ are defined as follows:

$$
x \oplus y=\min (1, x+y) \quad \neg x=1-x
$$

The derivate operations in $[0,1]_{M V}$ are given by $x \odot y=\max (0, x+y-1)$ (called Lukasiewicz t-norm) and $x \rightarrow y=\min (1,1-x+y)$. Finally the $M V$-lattice structure is the natural order in $[0,1]$.

A product $M V$-algebra $[22,23,24]$ (for short: $P M V$-algebra) is an algebra $\langle A, \oplus, \bullet, \neg, 0\rangle$ of type $\langle 2,2,1,0\rangle$ satisfying the following: 
$1\langle A, \oplus, \neg, 0\rangle$ is an $M V$-algebra,

$2\langle A, \bullet, 1\rangle$ is an abelian monoid,

$3 x \bullet(y \odot \neg z)=(x \bullet y) \odot \neg(x \bullet z)$.

An important example of $P M V$-algebra is $[0,1]_{M V}$ equipped with the usual multiplication (called product t-norm). This algebra is denoted by $[0,1]_{P M V}$. The following are almost immediate consequences of the definition of $P M V$-algebras:

Lemma 2.1 In each PMV-algebra we have
1. $0 \bullet x=0$,
2. If $a \leq b$ then $a \bullet x \leq b \bullet x$,
3. $x \odot y \leq x \bullet y \leq x \wedge y$.

Proposition 2.2 [23, Lemma 2.3] Each PMV-algebra is isomorphic to a subdirect product of linearly ordered PMV-algebras.

Definition 2.3 A $P M V_{\frac{1}{2^{4}}}$-algebra is an algebra $\left\langle A, \oplus, \bullet, \neg, 0, \frac{1}{2}, 1\right\rangle$ of type $\langle 2,2,1,0,0,0\rangle$ satisfying the following:

1. $\langle A, \oplus, \bullet, \neg, 0,1\rangle$ is a $P M V$-algebra,

2. $\neg \frac{1}{2}=\frac{1}{2}$,

3. $\frac{1}{2^{4}} \oplus \frac{1}{2^{4}}=\frac{1}{2^{3}} \quad$ where $\frac{1}{2^{n}}$ means the term $\frac{1}{2^{n-1}} \bullet \frac{1}{2}(n \geq 2)$.

It is well known that a $P M V$-algebra has at most a fix point of the negation [15, Lemma 2.10]. An example of $P M V_{\frac{1}{2^{4}}}$-algebra is $[0,1]_{P M V}$ where the fix point of the negation is $\frac{1}{2}$.

We denote $\mathcal{P} \mathcal{M V} \mathcal{1}_{2^{4}}$ the variety of $P M V_{\frac{1}{2^{4}}}$-algebras. This variety plays a crucial role in Section 5 and Section 7. 


\section{The Poincaré irreversible quantum gates system}

The Poincaré irreversible quantum computational system is framed in the model of quantum computation with mixed states. It takes into account a set of quantum gates -represented by quantum operations- acting on quantum mixed states -represented by density operators of $\mathcal{D}\left(\mathbb{C}^{2}\right)$. We first describe some basic properties of density operators in $\mathcal{D}\left(\mathbb{C}^{2}\right)$. Due to the fact that the Pauli matrices:

$$
\sigma_{x}=\left(\begin{array}{cc}
0 & 1 \\
1 & 0
\end{array}\right) \quad \sigma_{y}=\left(\begin{array}{cc}
0 & -i \\
i & 0
\end{array}\right) \quad \sigma_{z}=\left(\begin{array}{cc}
1 & 0 \\
0 & -1
\end{array}\right)
$$

plus $I$, where $I$ is the $2 \times 2$ identity matrix, are a basis for the set of operators over $\mathbb{C}^{2}$, an arbitrary density operator $\rho \in \mathcal{D}\left(\mathbb{C}^{2}\right)$ may be represented as

$$
\rho=\frac{1}{2}\left(I+r_{1} \sigma_{x}+r_{2} \sigma_{y}+r_{3} \sigma_{z}\right)
$$

where $r_{1}, r_{2}, r_{3}$ are real numbers such that $r_{1}^{2}+r_{2}^{2}+r_{3}^{2} \leq 1$. When a density operator $\rho \in \mathcal{D}\left(\mathbb{C}^{2}\right)$ represents a pure state, it can be identified with a point $\left(r_{1}, r_{2}, r_{3}\right)$ on the sphere of radius 1 (the Bloch sphere) and each $\rho \in \mathcal{D}\left(\mathbb{C}^{2}\right)$ that represents a mixed state with a point in the interior of the Bloch sphere. We denote this identifications as $\rho=\left(r_{1}, r_{2}, r_{3}\right)$. In this way $P_{1}=(0,0-1)$ and $P_{0}=(0,0,1)$. An interesting feature of density operators in $\mathcal{D}\left(\mathbb{C}^{2}\right)$ is the following: any real number $\lambda \in[0,1]$, uniquely determines a density operator $\rho_{\lambda}$ given by

$$
\rho_{\lambda}=(1-\lambda) P_{0}+\lambda P_{1}
$$

Lemma 3.1 $\left[6\right.$, Lemma 6.1] Let $\rho=\left(r_{1}, r_{2}, r_{3}\right) \in \mathcal{D}\left(\mathbb{C}^{2}\right)$. Then we have:

1. $p(\rho)=\frac{1-r_{3}}{2}$.

2. If $\rho=\rho_{\lambda}$ for some $\lambda \in[0,1]$ then $\rho=(0,0,1-2 \lambda)$ and $p\left(\rho_{\lambda}\right)=\lambda$.

Definition 3.2 The Poincaré irreversible quantum computational system $(\mathbb{I} \text {-system })_{3}[3,5]$ is given by the following set of quantum gates

- $\sigma \oplus \tau=\rho_{p(\sigma) \oplus p(\tau)}$

[Eukasiewicz gate]

- $\sigma \bullet \tau=\rho_{p(\sigma) \cdot p(\tau)}$

[IAND gate] 
- $\neg \tau=\sigma_{x} \tau \sigma_{x}^{\dagger}$

[NOT gate]

- $\sqrt{\tau}=\left(\begin{array}{cc}\frac{1+i}{2} & \frac{1-i}{2} \\ \frac{1-i}{2} & \frac{1+i}{2}\end{array}\right) \tau\left(\begin{array}{ll}\frac{1+i}{2} & \frac{1-i}{2} \\ \frac{1-i}{2} & \frac{1+i}{2}\end{array}\right)^{\dagger} \quad[\sqrt{N O T}$ gate $]$

where $\sigma$ and $\tau$ belong to $\mathcal{D}\left(\mathbb{C}^{2}\right)$.

By the Kraus representation mentioned in Section 1, it is immediate that $\neg$ and $\sqrt{ }$. are quantum operations. We refer to [8] for a representation of the IAND gate and the Lukasiewicz gate as quantum operations. The $\mathbb{I P}$-system defines a set of operations on $\mathcal{D}\left(\mathbb{C}^{2}\right)$ giving rise to the structure

$$
\left\langle\mathcal{D}\left(\mathbb{C}^{2}\right), \oplus, \bullet, \neg, \sqrt{ }, P_{0}, \rho_{\frac{1}{2}}, P_{1}\right\rangle
$$

known as Poincaré irreversible quantum computational algebra (shortly IQCalgebra) $[3,5]$. The following lemma gives the main properties of the $I Q C$ algebra.

Lemma 3.3 [6, Lemma 6.1] and [7, Lemma 3.7] Let $\tau, \sigma \in \mathcal{D}\left(\mathbb{C}^{2}\right)$ and let $p$ be the probability function over $\mathcal{D}\left(\mathbb{C}^{2}\right)$. Then we have:

1. $\left\langle\mathcal{D}\left(\mathbb{C}^{2}\right), \bullet\right\rangle$ and $\left\langle\mathcal{D}\left(\mathbb{C}^{2}\right), \oplus\right\rangle$ are abelian monoids,

2. $\tau \bullet P_{0}=P_{0}$,

3. $\tau \bullet P_{1}=\rho_{p(\tau)}$,

4. $p(\tau \bullet \sigma)=p(\tau) p(\sigma)$,

5. $p(\tau \oplus \sigma)=p(\tau) \oplus p(\sigma)$,

6. $\sqrt{\neg \tau}=\neg \sqrt{\tau}$,

7. $\sqrt{\sqrt{\tau}}=\neg \tau$.

Moreover if $\sigma=\left(r_{1}, r_{2}, r_{3}\right)$ then

8. $\neg \sigma=\left(r_{1},-r_{2},-r_{3}\right)$ and $\sqrt{\sigma}=\left(r_{1},-r_{3}, r_{2}\right)$, hence $p(\neg \sigma)=\frac{1+r_{3}}{2}$ and $p(\sqrt{\sigma})=\frac{1-r_{2}}{2}$,

9. $p(\sqrt{\tau \bullet \sigma})=p(\sqrt{\tau \oplus \sigma})=\frac{1}{2}$. 
Taking into account Lemma 3.3-7, $\neg$ becomes a definable operation in the $I Q C$-algebra. Recalling that in our case the assignment of probability is done via a function $p: \mathcal{D}\left(\mathbb{C}^{2}\right) \rightarrow[0,1]$, it is possible to establish the following equivalence relation in $\mathcal{D}\left(\mathbb{C}^{2}\right)$ :

$$
\sigma \equiv \tau \quad \text { iff } \quad p(\sigma)=p(\tau)
$$

This equivalence is strongly related to the preorder $\leq_{w}$ mentioned at the end of Section 1. In view of Lemma 3.1 and Lemma 3.3, we can see that $\equiv$ is a $(\oplus, \bullet, \neg)$-congruence but not a $\sqrt{ }$--congruence.

Proposition 3.4 Let $[\sigma]$ be the equivalence class of $\sigma \in \mathcal{D}\left(\mathbb{C}^{2}\right)$ and consider the natural application $\pi_{\equiv}: \mathcal{D}\left(\mathbb{C}^{2}\right) \rightarrow \mathcal{D}\left(\mathbb{C}^{2}\right) / \equiv$. Then

1. $[\sigma]=\left[\sigma \bullet P_{1}\right]=\left[\sigma \oplus P_{0}\right]=\left[\rho_{p(\sigma)}\right]$

2. $\left\langle\mathcal{D}\left(\mathbb{C}^{2}\right) / \equiv, \oplus, \bullet, \neg,\left[P_{0}\right],\left[\rho_{\frac{1}{2}}\right],\left[P_{1}\right]\right\rangle$ is a $P M V_{\frac{1}{2^{4}}}$-algebra and the assignment $\left[\rho_{\lambda}\right] \stackrel{f}{\rightarrow} \lambda$ is a $\mathcal{P} \mathcal{M} \mathcal{V}_{\frac{1}{2^{4}}}$-isomorphism from $\mathcal{D}\left(\mathbb{C}^{2}\right) / \equiv$ onto $[0,1]_{P M V}$.

3. The following diagram is commutative

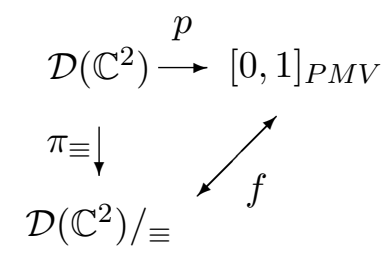

Proof: 1) Follows from Lemma 3.3. 2) By item 1, we can consider the identification $\left(\mathcal{D}\left(\mathbb{C}^{2}\right) / \equiv\right)=\left(\rho_{\lambda}\right)_{\lambda \in[0,1]}$. Then it may be easily proved that $\left\langle\left(\mathcal{D}\left(\mathbb{C}^{2}\right) / \equiv\right), \oplus, \bullet, \neg,\left[P_{0}\right],\left[\rho_{\frac{1}{2}}\right],\left[P_{1}\right]\right\rangle$ is a $P M V_{\frac{1}{2^{4}}}$-algebra and $\left[\rho_{\lambda}\right] \stackrel{f}{\rightarrow} \lambda$ is a $\mathcal{P} \mathcal{M V}{\frac{1}{2^{4}}}$-isomorphism from $\mathcal{D}\left(\mathbb{C}^{2}\right) / \equiv$ onto $[0,1]_{P M V}$. 3) Immediate.

Remark 3.5 By Proposition 3.4-3, we can see that the assignment of probability $p: \mathcal{D}\left(\mathbb{C}^{2}\right) \rightarrow[0,1]$ can be identified with the natural application $\pi_{\equiv}$ : $\mathcal{D}\left(\mathbb{C}^{2}\right) \rightarrow \mathcal{D}\left(\mathbb{C}^{2}\right) / \equiv$ where $\mathcal{D}\left(\mathbb{C}^{2}\right) / \equiv$ is endowed with a $\mathcal{P} \mathcal{M} \mathcal{V}_{\frac{1}{2^{4}}}$-structure. This crucial fact is particularly relevant in the definition of probabilistic consequence introduced in Section 6. 


\section{Quantum computational algebras}

In this Section we introduce algebraic structures by means of simple equations in an attempt to capture the basic properties of the $I Q C$-algebra.

The first and more basic algebraic structure associated to the $\mathbb{I P}$-system was introduced in [21] for the Eukasiewicz and the NOT gates. This is the quasi $M V$-algebra or $q M V$-algebra for short. A $q M V$-algebra is an algebra $\langle A, \oplus, \neg, 0,1\rangle$ of type $\langle 2,1,0,0\rangle$ satisfying the following equations:

Q1. $x \oplus(y \oplus z)=(x \oplus y) \oplus z$,

Q2. $\neg \neg x=x$,

Q3. $x \oplus 1=1$,

Q4. $\neg(\neg x \oplus y) \oplus y=\neg(\neg y \oplus x) \oplus x$,

Q5. $\neg(x \oplus 0)=\neg x \oplus 0$,

Q6. $(x \oplus y) \oplus 0=x \oplus y$,

Q7. $\neg 0=1$.

From an intuitive point of view, a $q M V$-algebra can be seen as an $M V$ algebra which fails to satisfy the equation $x \oplus 0=x$. We define the binary operations $\odot, \vee, \wedge, \rightarrow$ in the same way as we $\operatorname{did}$ for $M V$-algebras.

Lemma 4.1 ([21, Lemma 6]) The following equations are satisfied in each $q M V$-algebra:
1. $x \oplus y=y \oplus x$,
5. $x \oplus 0=x \wedge x$,
2. $x \oplus \neg x=1$,
6. $x \wedge y=y \wedge x$
3. $x \odot \neg x=0$,
7. $x \vee y=y \vee x$,
4. $0 \oplus 0=0$.

Another algebraic structure associated to the $\mathbb{I P}$-system was introduced

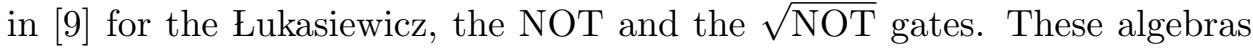
are known as square root quasi $M V$-algebras or $\sqrt{q M V}$-algebras for short. 
A $\sqrt{q M V}$-algebra is an algebra $\left\langle A, \oplus, \sqrt{,} 0, \frac{1}{2}, 1\right\rangle$ of type $\langle 2,1,0,0,0\rangle$ such that, upon defining $\neg x=\sqrt{\sqrt{x}}$ for all $x \in A$, the following conditions are satisfied:

SQ1. $\left\langle A, \oplus, \neg, 0, \frac{1}{2}, 1\right\rangle$ is a $q M V$-algebra,

SQ2. $\sqrt{\neg x}=\neg \sqrt{x}$,

SQ3. $\sqrt{x \oplus y} \oplus 0=\sqrt{\frac{1}{2}}=\frac{1}{2}$.

In what follows we will extend the $\sqrt{q M V}$-algebra structure taking into account the basic properties of the IAND gate given in Lemma 3.3.

Definition 4.2 A $\sqrt{q P M V}$-algebra is an algebra $\left\langle A, \oplus, \bullet, \sqrt{,} 0, \frac{1}{2}, 1\right\rangle$ of type $\langle 2,2,1,0,0,0\rangle$ satisfying the following:
1. $\left\langle A, \oplus, \sqrt{ }, 0, \frac{1}{2}, 1\right\rangle$ is a $\sqrt{q M V}$-algebra,
2. $x \bullet y=y \bullet x$,
3. $x \bullet(y \bullet z)=(x \bullet y) \bullet z$,
4. $x \bullet 1=x \oplus 0$,
5. $x \bullet y=(x \bullet y) \oplus 0$,
6. $x \bullet(y \odot \neg z)=(x \bullet y) \odot \neg(x \bullet z)$,
7. $\sqrt{x \bullet y} \oplus 0=\frac{1}{2}$.

We denote by $\sqrt{q \mathcal{P M \mathcal { V }}}$ the variety of $\sqrt{q P M V}$-algebras. It is not very hard to see that the $I Q C$-algebra $\left\langle\mathcal{D}\left(\mathbb{C}^{2}\right), \oplus, \bullet, \neg, \sqrt{ }, P_{0}, \rho_{\frac{1}{2}}, P_{1}\right\rangle$ is a $\sqrt{q P M V}$-algebra.

Let $A$ be a $\sqrt{q P M V}$-algebra. Then we define two binary relations $\leq$ and $\equiv$ on $A$ as follows:

$$
\begin{gathered}
a \leq b \text { iff } 1=a \rightarrow b \\
a \equiv b \text { iff } a \leq b \text { and } b \leq a
\end{gathered}
$$

Clearly, $\langle A, \leq\rangle$ is a preorder and one can also easily prove that $a \leq b$ iff $a \wedge b=a \oplus 0$ iff $a \vee b=b \oplus 0$. Moreover $a \equiv a \oplus 0$. 
Proposition 4.3 Let $A$ be $a \sqrt{q P M V}$-algebra and $a, b \in A$. Then:
1. $a \bullet 0=0$,
2. If $a \bullet b=1$ then $a \oplus 0=b \oplus 0=1$,
3. If $a \leq b$ then $a \bullet x \leq b \bullet x$,
4. $x \bullet y \leq x$,
5. $x \bullet(y \oplus 0)=(x \bullet y) \oplus 0$,
6. $\frac{1}{2}=\neg \frac{1}{2}$,
7. $\frac{1}{2} \oplus 0=\frac{1}{2}$,
8. $\sqrt{x \oplus y} \oplus \sqrt{z \oplus w}=1$.

Proof: $\quad 1) a \bullet 0=a \bullet(0 \odot \neg 0)=(a \bullet 0) \odot \neg(a \bullet 0)=0 . \quad 2)$ Suppose that $a \bullet b=1$. Then $\neg(a \oplus 0)=1 \odot \neg(a \bullet 1)=(a \bullet b) \odot \neg(a \bullet 1)=a \bullet(b \odot \neg 1)=0$. Thus $\neg(a \oplus 0)=0$, hence $a \oplus 0=1 . \quad 3)$ If $a \leq b$ then $1=a \rightarrow b=\neg(a \odot \neg b)$ and $0=a \odot \neg b$. Using item 1 . we have that $0=x \bullet 0=x \bullet(a \odot \neg b)=$ $(x \bullet a) \odot \neg(x \bullet b)$. Thus, $1=\neg((x \bullet a) \odot \neg(x \bullet b))=(x \bullet a) \rightarrow(x \bullet b)$ resulting $(x \bullet a) \leq(x \bullet b)$. 4) Since $x \leq 1$ by item 3 . we have that $x \bullet y \leq x \bullet 1=x \oplus 0 \leq x . \quad 5) x \bullet(y \oplus 0)=x \bullet(y \bullet 1)=(x \bullet y) \bullet 1=(x \bullet y) \oplus 0$. Items $6 ., 7$. and 8 . can be easily proved.

Definition 4.4 Let $A$ be a $\sqrt{q P M V}$-algebra. An element $a \in A$ is regular iff $a \oplus 0=a$. We denote by $\operatorname{Reg}(A)$ the set of regular elements.

Proposition 4.5 Let $A$ be a $\sqrt{q P M V}$-algebra. Then:

1. $\langle\operatorname{Reg}(A), \oplus, \bullet, \neg, 0,1\rangle$ is a $P M V$-algebra.

2. $\equiv$ is a $\langle\oplus, \bullet, \neg\rangle$-congruence on $A$ and $\langle A / \equiv, \oplus, \bullet, \neg,[0],[1]\rangle$ is a $P M V$ algebra.

3. $A / \equiv$ is $\mathcal{P} \mathcal{M V}$-isomorphic to $\operatorname{Reg}(A)$. This isomorphism is given by the assignment $[x] \mapsto x \oplus 0$. 
Proof: 1) From [21, Lemma 9] $\langle\operatorname{Reg}(A), \oplus, \neg, 0,1\rangle$ is an $M V$-algebra. Using Proposition 4.3-5, the operation • is closed in $\operatorname{Reg}(A)$. Now from the axioms of the $\sqrt{q P M V}$-algebras, $\langle\operatorname{Reg}(A), \oplus, \bullet, \neg, 0,1\rangle$ results a $P M V$ algebra.

2) It is easy to see that $\equiv$ is a $\langle\oplus, \neg\rangle$-congruence and $\langle A / \equiv, \oplus, \neg,[0]\rangle$ is an $M V$-algebra. For technical details see [21]. By Proposition 4.3-3, $\equiv$ is compatible with $\bullet$. Note that $[1]$ is the identity in $\langle A / \equiv, \bullet,[1]\rangle$ since $[x] \bullet[1]=[x \bullet 1]=[x \oplus 0]=[x]$. Hence, by definition of $\sqrt{q P M V}$-algebra, $\langle A / \equiv, \oplus, \bullet, \neg,[0],[1]\rangle$ is a $P M V$-algebra.

3) Since $[x]=[x \oplus 0]$ for each $x \in A$, then $\varphi$ is injective. If $x \in \operatorname{Reg}(A)$ then $x=x \oplus 0$. Therefore $\varphi([x])=x \oplus 0=x$ and $\varphi$ is surjective. Using Proposition 4.3-5 we have that $\varphi([x] \bullet[y])=\varphi([x \bullet y])=(x \bullet y) \oplus 0=$ $(x \oplus 0) \bullet(y \oplus 0)=\varphi([x]) \bullet \varphi([y])$. In the same way we can prove that $\varphi([x] \oplus[y])=\varphi([x]) \oplus \varphi([y])$. By axiom Q5, $\varphi(\neg[x])=\neg \varphi([x])$ and $\varphi([c])=c$ for $c \in\left\{0, \frac{1}{2}, 1\right\}$ since they are regular elements in $A$. Thus $[x] \mapsto x \oplus 0$ is a $\mathcal{P} \mathcal{M V}$-isomorphism.

Definition 4.6 Let $\langle A, \oplus, \bullet, \neg, 0,1\rangle$ be a $P M V$-algebra with fix point of the negation $\frac{1}{2}$. The pair algebra over $A$ is the algebra

$$
S_{A}=\left\langle A \times A, \oplus, \bullet, \sqrt{ }, 0, \frac{1}{2}, 1\right\rangle
$$

where:

$$
\begin{array}{ll}
(a, b) \oplus(c, d):=\left(a \oplus c, \frac{1}{2}\right), & 0:=\left(0, \frac{1}{2}\right) \\
(a, b) \bullet(c, d):=\left(a \bullet c, \frac{1}{2}\right), & 1:=\left(1, \frac{1}{2}\right) \\
\sqrt{(a, b)}:=(b, \neg a), & \frac{1}{2}:=\left(\frac{1}{2}, \frac{1}{2}\right)
\end{array}
$$

Proposition 4.7 Let $A$ be a PMV-algebra with fix point of the negation $\frac{1}{2}$. Then:

1. The pair algebra $S_{A}$ is a $\sqrt{q P M V}$-algebra.

2. $(a, b) \leq(c, d)$ in $S_{A}$ iff $a \leq c$ in $A$.

3. $R\left(S_{A}\right)$ is $\mathcal{P} \mathcal{M V}$-isomorphic to $A$. 
Proof: 1) It is not very hard to see that $\left\langle A \times A, \oplus, \sqrt{ }, 0, \frac{1}{2}, 1\right\rangle$, where $\neg x=\sqrt{\sqrt{x}}$, is a $\sqrt{q M V}$-algebra. We only have to prove that $S_{A}$ satisfies axioms 6 and 7 of $\sqrt{q P M V}$-algebras.

Ax 6) $x \bullet(y \odot \neg z)=(x \bullet y) \odot \neg(x \bullet z)$. In fact, $(a, b) \bullet((c, d) \odot \neg(z, w))=$ $(a, b) \bullet\left((c, d) \odot(\neg z, \neg w)=\left(a \bullet(c \odot \neg z), \frac{1}{2}\right)=\left((a \bullet c) \odot \neg(a \bullet z), \frac{1}{2}\right)\right.$. On the other hand $((a, b) \bullet(c, d)) \odot \neg((a, b) \bullet(z, w))=\left(a \bullet c, \frac{1}{2}\right) \odot\left(\neg(a \bullet z), \frac{1}{2}\right)=$ $\left((a \bullet c) \odot \neg(a \bullet z), \frac{1}{2}\right)$.

Ax 7) $\sqrt{x \bullet y} \oplus 0=\frac{1}{2}$. In fact: $\sqrt{(a, b) \bullet(c, d)} \oplus\left(0, \frac{1}{2}\right)=\sqrt{\left(a \bullet c, \frac{1}{2}\right)} \oplus$ $\left(0, \frac{1}{2}\right)=\left(\frac{1}{2}, \neg(a \bullet c)\right) \oplus\left(0, \frac{1}{2}\right)=\left(\frac{1}{2}, \frac{1}{2}\right)=\frac{1}{2}$.

Hence $S_{A}$ is a $\sqrt{q P M V}$-algebra.

2) $(a, b) \leq(c, d)$ iff $\left(1, \frac{1}{2}\right)=(a, b) \rightarrow(c, d)=\left(\neg a \oplus b, \frac{1}{2}\right)$ iff $1=\neg a \oplus b$ in $A$ iff $a \leq b$ in $A$.

3) If we consider $S_{A} \oplus 0=\left\{(x, y) \oplus\left(0, \frac{1}{2}\right):(x, y) \in A \times A\right\}$ then we have that $S_{A} \oplus 0=\left\{\left(x, \frac{1}{2}\right): x \in A\right\}$. Therefore, $S_{A} \oplus 0$ is $\mathcal{P} \mathcal{M V}$-isomorphic to $A$. Then, by Proposition $4.5, R\left(S_{A}\right)$ is $\mathcal{P} \mathcal{M V}$-isomorphic to $A$.

Definition 4.8 A $\sqrt{q P M V}$-algebra is called flat iff it satisfies the equation $0=1$.

Note that if $A$ is flat $\sqrt{q P M V}$-algebra then $\operatorname{Reg}(A)=\{0\}$ and $x \oplus y=$ $x \bullet y=0$ for each $x, y \in A$. There is a standard technique for extracting a flat $\sqrt{q P M V}$-algebra out of an arbitrary $\sqrt{q P M V}$-algebra:

Let $\left\langle A, \oplus, \bullet, \sqrt{ }, 0, \frac{1}{2}, 1\right\rangle$ be $\sqrt{q P M V}$-algebra. Define the structure

$$
F l(A)=\left\langle A, \oplus, \bullet, \sqrt{ }, 0^{F}, \frac{1}{2}^{F}, 1^{F}\right\rangle
$$

where

1. $0^{F}=\frac{1}{2}^{F}=1^{F}$

2. $x \oplus y=x \bullet y=0^{F}$

3. $\sqrt{x}=x$

Such an algebra is easily seen to be a flat $\sqrt{q P M V}$-algebra. 
Proposition 4.9 Let $A$ be a PMV-algebra with fix point of the negation $\frac{1}{2}$ and $F$ be a flat $\sqrt{q P M V}$-algebra that satisfies $\sqrt{x}=x$. Consider the algebra $D=F \times S$ where $S$ is a sub $\sqrt{q P M V}$-algebra of the pair algebra $S_{A}$. Then

1. $\left\{\left(0,\left(r_{2}, r_{3}\right)\right):\left(0,\left(r_{2}, r_{3}\right)\right) \in D\right\}$ defines a sub $\sqrt{q P M V}$-algebra of $D$, $\sqrt{q \mathcal{P M V}}$-isomorphic to $S$.

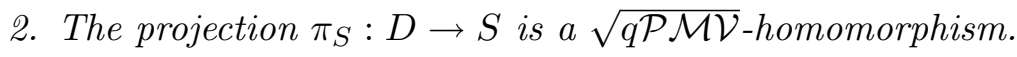

3. If $t$ contains an occurrence of $\star \in\{\oplus, \bullet\}$ then only one of the following possibilities holds: for each D-valuation $v, v(t)=\left(0, r, \frac{1}{2}\right)$ or for each $D$-valuation $v, v(t)=\left(0, \frac{1}{2}, r\right)$.

4. If $t$ contains an occurrence of $\star \in\{\oplus, \bullet\}$ then, for each $D$-valuation $v$, there exists a $S$-valuation $v^{\prime}$ such that $v(t)=v^{\prime}(t)$.

5. $D \models t=s$ iff $S \models t=s$

Proof: 1,2) are straightforward. In what follows we identify $(x,(y, z))$ with $(x, y, z)$ for each $(x,(y, z)) \in D$.

$3)$ Induction on the complexity of $t$. Since $t$ contains at least an occurrence of $\star \in\{\oplus, \bullet\}$, it cannot be an atomic term. Its minimum possible complexity is therefore represented by the case in which $t$ is $t_{1} \star t_{2}$ where each $t_{i}$ is either a variable or constant. Thus for each $D$-valuation $v$, $v(t)=v\left(t_{1} \star t_{2}\right)=\left(0, r, \frac{1}{2}\right)$ for some $r \in A$. Now let our claim hold whenever the complexity of a term is less that $n$ and $t$ has complexity $n$. Suppose that $t$ is $\sqrt{s}$. If $v(s)=\left(0, r, \frac{1}{2}\right)$ then $v(\sqrt{t})=\sqrt{\left(0, r, \frac{1}{2}\right)}=\left(0, \frac{1}{2}, \neg r\right)$. If $v(s)=\left(0, \frac{1}{2}, r\right)$ then $v(\sqrt{t})=\sqrt{\left(0, \frac{1}{2}, r\right)}=\left(0, r, \frac{1}{2}\right)$. The case in which $t$ is $s_{1} \star s_{2}$ is immediate.

4) Let $v$ be a $D$-valuation. By item 2, the composition $v^{\prime}=\pi_{S} v$ is

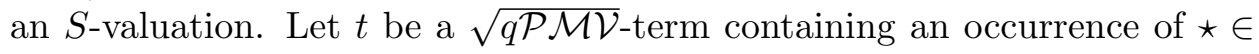
$\{\oplus, \bullet\}$. By item 3 , for each $D$-valuation $v, v(t)=\left(0, r_{2}, r_{3}\right)=\pi_{S}\left(0, r_{2}, r_{3}\right)=$ $\pi_{S} v(t)=v^{\prime}(t)$.

5) We will consider the non trivial direction. Suppose that $D \not \forall t=s$. If neither $t$ nor $s$ contain any occurrence of $\oplus$, then $t$ is ${\sqrt{t_{1}}}^{n}$ and $s$ is ${\sqrt{s_{1}}}^{m}$, where $n, m \geq 1$ are indexes of the successive application of the operation. If $t_{1}$ is a constant symbol then trivially $S \not \models t=s$ since $s_{1}$ is a variable or constant symbol. If $t_{1}$ and $s_{1}$ are both variables then $D \not \forall t=s$ implies that $t_{1}$ and $t_{2}$ are different. Hence evaluate $t_{1}$ to $\left(0,0, \frac{1}{2}\right)$ and $s_{1}$ to $\left(0, \frac{1}{2}, \frac{1}{2}\right)$ to get the required counterexample. 
Suppose that $t$ contains an occurrence of $\star \in\{\oplus, \bullet\}$ but $s$ does not. We have to consider the following three sub cases:

i) If $s$ is a constant symbol then $D \models s=s \oplus 0$ and $D \not \models t=s \oplus 0$. Let $v$ be a $D$-valuation such that $v(t) \neq v(s \oplus 0)$. By item 4 , there exists an $S$-valuation $v^{\prime}$ such that $v(t)=v^{\prime}(t)$ and $v(s \oplus 0)=v^{\prime}(s \oplus 0)$. Thus $v^{\prime}(t) \neq v^{\prime}(s \oplus 0)=v^{\prime}(s)$ and $S \not \models t=s$.

ii) Suppose that $s$ is a variable. By item 3, we first assume that $v(t)$ has the form $\left(0, r, \frac{1}{2}\right)$ for each $S$-valuation $v$. Then evaluate $s$ to $(0,1,0)$ to get the required counterexample. If we assume that $v(t)$ has the form $\left(0, \frac{1}{2}, r\right)$ for each $D$-valuation $v$, evaluate $s$ to $(0,0,1)$ to get the required counterexample. Hence $S \not \models t=s$.

iii) With the same argument used in ii, we can prove that $S \not \models t=s$ when $s$ is ${\sqrt{s_{1}}}^{m}$.

Suppose that $t$ and $s$ contain an occurrence of $\star \in\{\oplus, \bullet\}$. Since $D \not \models$ $t=s$ then there exists a $D$-valuation $v$ such that $v(t) \neq v(s)$. By item 4, there exists an $S$-valuation $v^{\prime}$ such that $v(t)=v^{\prime}(t)$ and $v(s)=v^{\prime}(s)$. Hence $v^{\prime}(t) \neq v^{\prime}(s)$ and $S \not \models t=s$.

Proposition 4.10 Let $A$ be a $\sqrt{q P M V}$-algebra. Consider the $\sqrt{q P M V}$ algebra $A^{*}=F l(A) \times S_{\operatorname{Reg}(A)}$ and the application $f: A \rightarrow A^{*}$ such that

$$
f(x)= \begin{cases}(0,(x \oplus 0, \sqrt{x} \oplus 0)), & \text { if } x \in \operatorname{Reg}(A) \\ (x,(x \oplus 0, \sqrt{x} \oplus 0)), & \text { if } x \notin \operatorname{Reg}(A)\end{cases}
$$

Then $f$ is an injective $\sqrt{q \mathcal{P M V}}$-homomorphism.

Proof: $\quad$ By definition $f$ is an injective function. We need to prove that $f$

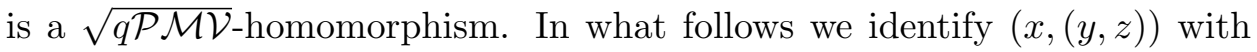
$(x, y, z)$ for each $(x,(y, z)) \in A^{*}$.

- Let $a \in\left\{0, \frac{1}{2}, 1\right\}$. In this case $a \in \operatorname{Reg}(A)$ and $\sqrt{a} \oplus 0=\frac{1}{2}$. Therefore $f(a)=(0, a \oplus 0, \sqrt{a} \oplus 0)=\left(0, a, \frac{1}{2}\right)$.

- Let $\star \in\{\oplus, \bullet\} . f(x \star y)=(0,(x \star y) \oplus 0, \sqrt{x \star y} \oplus 0)=\left(0,(x \star y) \oplus 0, \frac{1}{2}\right)=$ $\left(t_{x}, x \oplus 0, \sqrt{x} \oplus 0\right) \star\left(t_{y}, y \oplus 0, \sqrt{y} \oplus 0\right)=f(x) \star f(y)$.

- For each $x \in A$, we define $t_{x}$ as follows:

$$
t_{x}= \begin{cases}0, & \text { if } x \in \operatorname{Reg}(A) \\ x, & \text { if } x \notin \operatorname{Reg}(A)\end{cases}
$$




$$
\begin{aligned}
& \text { Then } f(\sqrt{x})=\left(t_{\sqrt{x}}, \sqrt{x} \oplus 0, \sqrt{\sqrt{x}} \oplus 0\right)=\left(t_{\sqrt{x}}, \sqrt{x} \oplus 0, \neg(x \oplus 0)\right)= \\
& \sqrt{\left(t_{\sqrt{x}}, x \oplus 0, \sqrt{x} \oplus 0\right)}=\sqrt{f(x)} .
\end{aligned}
$$

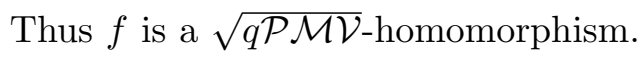

Theorem 4.11 Let $\mathcal{S}^{\square}$ be the sub-class of pair algebras $S_{A}$ where $A$ is a $P M V$-chain with fix point of the negation. Then

$$
\sqrt{q \mathcal{P} \mathcal{M V}}=\mathcal{V}\left(\mathcal{S}^{\square}\right)
$$

Proof: $\quad$ We shall prove that $\sqrt{q \mathcal{P \mathcal { M V }}}=t=s$ iff $\mathcal{S}^{\square} \models t=s$. As regards the non-trivial direction, assume that $\mathcal{S}^{\square} \models t=s$. Suppose that there exists

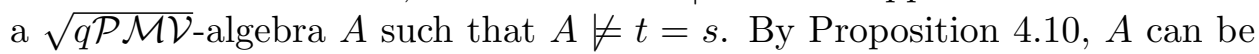
embedded in $F l(A) \times S_{R e g(A)}$. Therefore $F l(A) \times S_{R e g(A)} \not \neq t=s$ and by Proposition 4.9-5, $S_{\operatorname{Reg}(A)} \not \models t=s$. By Proposition 2.2 we can consider a subdirect representation $\beta: \operatorname{Reg}(A) \hookrightarrow \Pi_{i \in I} A_{i}$ such that $\left(A_{i}\right)_{i \in I}$ is a family of $P M V$-chains. For each $i \in I$, let $p_{i}$ be the $i$ th-projection in $A_{i}$ and consider the following function:

$$
\beta_{i}: S_{R e g(A)} \rightarrow S_{A_{i}} \quad \text { s.t. } \quad(x, y) \mapsto \beta_{i}((x, y))=\left(p_{i} \beta(x), p_{i} \beta(y)\right)_{i \in I}
$$

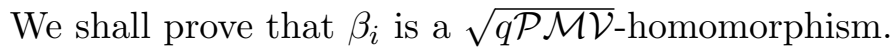

- The preservation of $\left(0, \frac{1}{2}\right),\left(\frac{1}{2}, \frac{1}{2}\right)$ and $\left(1, \frac{1}{2}\right)$ is immediate.

- Let $\star \in\{\oplus, \bullet\} . \beta_{i}\left(\left(x_{1}, y_{1}\right) \star\left(x_{2}, y_{2}\right)\right)=\beta_{i}\left(x_{1} \star x_{2}, \frac{1}{2}\right)=\left(x_{1 i} \star x_{2 i}, \frac{1}{2}{ }_{i}\right)=$ $\left(x_{1 i}, y_{1 i}\right) \star\left(x_{2 i}, y_{2 i}\right)=\beta_{i}\left(\left(x_{1}, y_{1}\right)\right) \star \beta_{i}\left(\left(x_{2}, y_{2}\right)\right)$.

- $\beta_{i}(\sqrt{(x, y)})=\beta_{i}((y, \neg x))=\left(y_{i}, \neg x_{i}\right)=\sqrt{\left(x_{i}, y_{i}\right)}=\sqrt{\beta_{i}(x, y)}$.

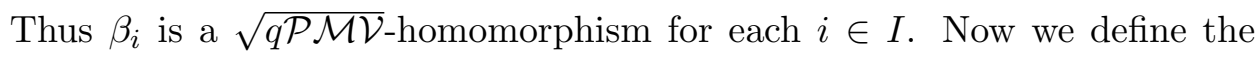
function

$$
\beta^{*}: S_{\operatorname{Reg}(A)} \rightarrow \Pi_{i \in I} S_{A_{i}} \quad \text { s.t. }(x, y) \mapsto \beta^{*}((x, y))=\left(\beta_{i}(x, y)\right)_{i \in I}
$$

Note that $\beta^{*}$ is injective since $\beta$ is a subdirect embedding. Moreover $\beta^{*}$ is a $\sqrt{q \mathcal{P M V}}$-homomorphism since $\beta_{i}$ is a $\sqrt{q \mathcal{P} \mathcal{M V}}$-homomorphism for each $i \in I$. Thus $S_{\operatorname{Reg}(A)} \not \neq t=s$ implies that there exists $m \in I$ such that $S_{A_{m}} \not$ $t=s$ which is contradiction since $S_{A_{m}}$ lies in $\mathcal{S}^{\square}$. Hence $\sqrt{q \mathcal{P \mathcal { M V }}}=t=s$. 
We have seen that the structure of the $\sqrt{q P M V}$-algebra is a good abstraction for the $I Q C$-algebra. However, we do not yet know whether $\sqrt{q \mathcal{P M V}}=\mathcal{V}\left(\mathcal{D}\left(\mathbb{C}^{2}\right)\right)$. In what follows we will show that this is not the case. In order to do it, we need some preliminary results:

Lemma 4.12 1. The set $\mathcal{D}\left(\mathbb{C}^{2}\right)_{y, z}=\left\{(0, y, z):(0, y, z) \in \mathcal{D}\left(\mathbb{C}^{2}\right)\right\}$ defines a sub $\sqrt{q P M V}$-algebra of $\mathcal{D}\left(\mathbb{C}^{2}\right)$.

2. Consider the real interval $[-1,1]$ equipped with the following operations: $x \oplus y=x \bullet y=0, \sqrt{x}=x$ and $0^{[-1,1]}=\frac{1}{2}^{[-1,1]}=1^{[-1,1]}=0$. Then $F_{[-1,1]}=\left\langle[-1,1], \oplus, \bullet, \sqrt{ }, 0^{[-1,1]}, \frac{1}{2}^{[-1,1]}, 1^{[-1,1]}\right\rangle$ is a flat algebra.

3. $f: F_{[-1,1]} \times \mathcal{D}\left(\mathbb{C}^{2}\right)_{y, z} \rightarrow \mathcal{D}\left(\mathbb{C}^{2}\right)$ such that $(x,(0, y, z)) \mapsto(x, y, z)$ is a $\sqrt{q P M V}$-isomorphism.

Proof: Straightforward.

Let $S_{[0,1]}$ be the pair algebra over the standard $P M V$-algebra $[0,1]_{P M V}$. If we consider the set

$$
D_{[0,1]}=\left\{(x, y) \in S_{[0,1]}:\left(x-\frac{1}{2}\right)^{2}+\left(y-\frac{1}{2}\right)^{2} \leq \frac{1}{4}\right\}
$$

it is not very hard to see that $\left\langle D_{[0,1]}, \oplus, \bullet, \sqrt{ }, 0, \frac{1}{2}, 1\right\rangle$ is a sub $\sqrt{q P M V}$ algebra of $S_{[0,1]}$. Moreover $\operatorname{Reg}\left(D_{[0,1]}\right)=\operatorname{Reg}\left(S_{[0,1]}\right)$ and it is $P M V$-isomorphic to $[0,1]_{P M V}$.

Proposition 4.13 $\quad$ 1. $\varphi: \mathcal{D}\left(\mathbb{C}^{2}\right)_{y, z} \rightarrow D_{[0,1]}$ such that $\varphi(y, z)=\left(\frac{1-z}{2}, \frac{1-y}{2}\right)$ is a $\sqrt{q \mathcal{P M \mathcal { V }}}$-isomorphism.

2. $\mathcal{D}\left(\mathbb{C}^{2}\right) \models t=s$ iff $D_{[0,1]} \models t=s$.

Proof: 1) Let $\sigma=(0, b, c) \in \mathcal{D}\left(\mathbb{C}^{2}\right)_{y, z}$. Then $\varphi(\sigma)=\left(\frac{1-c}{2}, \frac{1-b}{2}\right)$ and $\left(\frac{1-c}{2}-\frac{1}{2}\right)^{2}+\left(\frac{1-b}{2}-\frac{1}{2}\right)^{2}=\frac{1}{4}\left(c^{2}+b^{2}\right) \leq \frac{1}{4}$. Thus the image of $\varphi$ is contained in $D_{[0,1]}$. It is clear that $\varphi$ is injective. Let $(a, b) \in D_{[0,1]}$. If we consider $\sigma=(0,1-2 b, 1-2 a)$ then $(1-2 b)^{2}+(1-2 a)^{2}=4\left(\frac{1}{2}-a\right)^{2}+4\left(\frac{1}{2}-b\right)^{2} \leq 1$. Hence $\sigma \in \mathcal{D}\left(\mathbb{C}^{2}\right)_{y, z}, \varphi(\sigma)=(a, b)$ and $\varphi$ is a surjective map. Now we prove

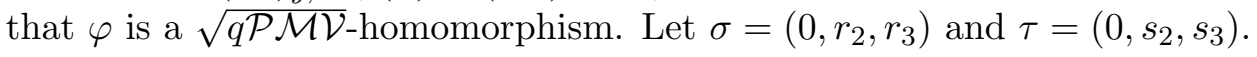
Using Lemma 3.1 and Lemma 3.3 we have that:

- Let $\star \in\{\oplus, \bullet\} . \varphi(\sigma \star \tau)=\varphi\left(\rho_{p(\sigma) \star p(\rho)}\right)=\varphi(0,0,1-2(p(\sigma) \star p(\rho)))=$ $\left(p(\sigma) \star p(\rho), \frac{1}{2}\right)=\left(\frac{1-r_{3}}{2}, \frac{1-r_{2}}{2}\right) \star\left(\frac{1-s_{3}}{2}, \frac{1-s_{2}}{2}\right)=\varphi(\sigma) \star \varphi(\tau)$. 
- $\varphi(\sqrt{\sigma})=\varphi\left(0,-r_{3}, r_{2}\right)=\left(\frac{1-r_{2}}{2}, \frac{1+r_{3}}{2}\right)=\left(\frac{1-r_{2}}{2}, 1-\frac{1-r_{3}}{2}\right)=\sqrt{\left(\frac{1-r_{3}}{2}, \frac{1-r_{2}}{2}\right)}=$ $\sqrt{\varphi(\sigma)}$.

- $\varphi\left(P_{1}\right)=\varphi(0,0,-1)=\left(1, \frac{1}{2}\right), \quad \varphi\left(P_{0}\right)=\varphi(0,0,1)=\left(0, \frac{1}{2}\right) \quad$ and $\varphi\left(\rho_{\frac{1}{2}}\right)=\varphi(0,0,0)=\left(\frac{1}{2}, \frac{1}{2}\right)$.

Thus $\varphi$ is $\sqrt{q \mathcal{P M V}}$-isomorphism.

2) By item 1 and Lemma 4.12 we can see that $\mathcal{D}\left(\mathbb{C}^{2}\right)$ is $\sqrt{q \mathcal{P M \mathcal { V }}}$ isomorphic to $F_{[-1,1]} \times D_{[0,1]}$. Hence by Proposition 4.9-5 $\mathcal{D}\left(\mathbb{C}^{2}\right) \models t=s$ iff $D_{[0,1]} \models t=s$.

Proposition 4.14 Consider the pair algebra $S_{[0,1]}$ and $a=\left(a_{1}, a_{2}\right) \in S_{[0,1]}$. Then the following conditions are equivalent:

1. $a \in D_{[0,1]}$

2. a satisfies the equation $1=\left(\frac{1}{2^{4}} \oplus\left(\frac{x \bullet x}{2^{2}} \oplus \frac{\sqrt{x} \bullet \sqrt{x}}{2^{2}}\right)\right) \rightarrow\left(\frac{x}{2^{2}} \oplus \frac{\sqrt{x}}{2^{2}}\right)$,

3. a satisfies the equation $1=\left(\frac{1}{2^{4}} \oplus\left(\frac{\neg x \bullet \neg x}{2^{2}} \oplus \frac{\sqrt{x} \bullet \sqrt{x}}{2^{2}}\right)\right) \rightarrow\left(\frac{\neg x}{2^{2}} \oplus \frac{\sqrt{x}}{2^{2}}\right)$,

4. a satisfies the equation $1=\left(\frac{1}{2^{4}} \oplus\left(\frac{x \bullet x}{2^{2}} \oplus \frac{\sqrt{\neg x} \bullet \sqrt{\neg x}}{2^{2}}\right)\right) \rightarrow\left(\frac{x}{2^{2}} \oplus \frac{\sqrt{\neg x}}{2^{2}}\right)$,

5. a satisfies the equation $1=\left(\frac{1}{2^{4}} \oplus\left(\frac{\neg x \bullet \neg x}{2^{2}} \oplus \frac{\sqrt{\neg x} \cdot \sqrt{\neg x}}{2^{2}}\right)\right) \rightarrow\left(\frac{\neg x}{2^{2}} \oplus \frac{\sqrt{\neg x}}{2^{2}}\right)$,

where $\frac{1}{2^{n}}$ means the term $\frac{1}{2^{n-1}} \bullet \frac{1}{2}$ and $\frac{t}{2^{n}}$ means the term $t \bullet \frac{1}{2^{n}}(n \geq 2)$.

Proof: $\quad 1 \Longleftrightarrow 2) 1=\left(\frac{1}{2^{4}} \oplus\left(\frac{x \bullet x}{2^{2}} \oplus \frac{\sqrt{x} \bullet \sqrt{x}}{2^{2}}\right)\right) \rightarrow\left(\frac{x}{2^{2}} \oplus \frac{\sqrt{x}}{2^{2}}\right)$ is satisfied by $a=$ $\left(a_{1}, a_{2}\right) \in S_{[0,1]}$ iff $\left(1, \frac{1}{2}\right)=\left(\left(\frac{1}{2^{4}}, \frac{1}{2}\right) \oplus\left(\left(\frac{a_{1}^{2}}{2^{2}}, \frac{1}{2}\right) \oplus\left(\frac{a_{2}^{2}}{2^{2}}, \frac{1}{2}\right)\right)\right) \rightarrow\left(\left(\frac{a_{1}}{2^{2}}, \frac{1}{2}\right) \oplus\left(\frac{a_{2}}{2^{2}}, \frac{1}{2}\right)\right)$ iff $\left(1, \frac{1}{2}\right)=\left(\frac{1}{2^{4}} \oplus \frac{a_{1}^{2}}{2^{2}} \oplus \frac{a_{2}^{2}}{2^{2}}, \frac{1}{2}\right) \rightarrow\left(\frac{a_{1}}{2^{2}} \oplus \frac{a_{2}}{2^{2}}, \frac{1}{2}\right)$ iff $1=\left(\frac{1}{2^{4}} \oplus \frac{a_{1}^{2}}{2^{2}} \oplus \frac{a_{2}^{2}}{2^{2}}\right) \rightarrow\left(\frac{a_{1}}{2^{2}} \oplus \frac{a_{2}}{2^{2}}\right)$ iff $\frac{1}{2^{4}} \oplus \frac{a_{1}^{2}}{2^{2}} \oplus \frac{a_{2}^{2}}{2^{2}} \leq \frac{a_{1}}{2^{2}} \oplus \frac{a_{2}}{2^{2}}$ iff $\frac{1}{2^{4}}+\frac{a_{1}^{2}}{2^{2}}+\frac{a_{2}^{2}}{2^{2}} \leq \frac{a_{1}}{2^{2}}+\frac{a_{2}}{2^{2}}$ iff $a_{1}^{2}-a_{1}+\frac{1}{2^{2}}+a_{2}^{2}-a_{2}+\frac{1}{2^{2}} \leq \frac{1}{2^{2}}$ iff $\left(a_{1}-\frac{1}{2}\right)^{2}+\left(a_{2}-\frac{1}{2}\right)^{2} \leq \frac{1}{4}$ iff $a=\left(a_{1}, a_{2}\right) \in D_{[0,1]}$.

$1 \Longleftrightarrow 3) 1=\left(\frac{1}{2^{4}} \oplus\left(\frac{\neg x \bullet \neg x}{2^{2}} \oplus \frac{\sqrt{x} \bullet \sqrt{x}}{2^{2}}\right)\right) \rightarrow\left(\frac{\neg x}{2^{2}} \oplus \frac{\sqrt{x}}{2^{2}}\right)$ is satisfied by $a=$ $\left(a_{1}, a_{2}\right) \in S_{[0,1]}$ iff $\left(1, \frac{1}{2}\right)=\left(\left(\frac{1}{2^{4}}, \frac{1}{2}\right) \oplus\left(\left(\frac{\left(1-a_{1}\right)^{2}}{2^{2}}, \frac{1}{2}\right) \oplus\left(\frac{a_{2}^{2}}{2^{2}}, \frac{1}{2}\right)\right)\right) \rightarrow\left(\left(\frac{1-a_{1}}{2^{2}}, \frac{1}{2}\right) \oplus\right.$ $\left.\left(\frac{a_{2}}{2^{2}}, \frac{1}{2}\right)\right)$ iff $\left(1, \frac{1}{2}\right)=\left(\frac{1}{2^{4}} \oplus \frac{\left(1-a_{1}\right)^{2}}{2^{2}} \oplus \frac{a_{2}^{2}}{2^{2}}, \frac{1}{2}\right) \rightarrow\left(\frac{\left(1-a_{1}\right)}{2^{2}} \oplus \frac{a_{2}}{2^{2}}, \frac{1}{2}\right)$ iff $1=\left(\frac{1}{2^{4}} \oplus\right.$ 
$\left.\frac{\left(1-a_{1}\right)^{2}}{2^{2}} \oplus \frac{a_{2}^{2}}{2^{2}}\right) \rightarrow\left(\frac{1-a_{1}}{2^{2}} \oplus \frac{a_{2}}{2^{2}}\right)$ iff $\frac{1}{2^{4}} \oplus \frac{\left(1-a_{1}\right)^{2}}{2^{2}} \oplus \frac{a_{2}^{2}}{2^{2}} \leq \frac{1-a_{1}}{2^{2}} \oplus \frac{a_{2}}{2^{2}}$ iff $\frac{1}{8}+\frac{a_{1}^{2}}{2}+\frac{a_{2}^{2}}{2} \leq$ $\frac{a_{1}}{2}+\frac{a_{2}}{2}$ iff $\left(a_{1}-\frac{1}{2}\right)^{2}+\left(a_{2}-\frac{1}{2}\right)^{2} \leq \frac{1}{4}$ iff $a=\left(a_{1}, a_{2}\right) \in D_{[0,1]}$.

With the same argument we can prove $1 \Longleftrightarrow 4)$ and $1 \Longleftrightarrow 5$ ).

Theorem $4.15 \sqrt{q \mathcal{P} \mathcal{M V}} \neq \mathcal{V}\left(\mathcal{D}\left(\mathbb{C}^{2}\right)\right)$

Proof: By Proposition 4.13-2 and Proposition 4.14, the equation $1=$ $\left(\frac{1}{16} \oplus\left(\frac{x \bullet x}{4} \oplus \frac{\sqrt{x} \bullet \sqrt{x}}{4}\right)\right) \rightarrow\left(\frac{x}{4} \oplus \frac{\sqrt{x}}{4}\right)$ holds in $\mathcal{D}\left(\mathbb{C}^{2}\right)$ and fails in $S_{[0,1]}$. Hence $\sqrt{q \mathcal{P} \mathcal{M V}} \neq \mathcal{V}\left(\mathcal{D}\left(\mathbb{C}^{2}\right)\right)$

\section{Poincaré irreversible algebras}

In this section we introduce and study an algebraic structure called "Poincaré irreversible algebra" with the following motivation. As shown by Proposition 4.13 the $\sqrt{q \mathcal{P M V}}$-equations which hold in the $I Q C$-algebra coincide

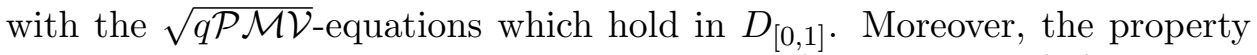
that characterizes $D_{[0,1]}$, i.e. a circle of radius $\frac{1}{2}$ and center $\left(\frac{1}{2}, \frac{1}{2}\right)$ circumscribed in the square $[0,1] \times[0,1]$, may be captured by the set of equations given in Proposition 4.14. Since these four equations may be formulated in the language of $\sqrt{q \mathcal{P} \mathcal{M V}}$, this allows to study a subvariety of the $\sqrt{q P M V}$ algebras that captures in a more faithful manner the basic properties of the $I Q C$-algebra.

Definition 5.1 A Poincaré irreversible algebra (IP-algebra for short) is a $\sqrt{q P M V}$-algebra satisfying the following axioms:

IP1. $1=\left(\frac{1}{2^{4}} \oplus\left(\frac{x \bullet x}{2^{2}} \oplus \frac{\sqrt{x} \bullet \sqrt{x}}{2^{2}}\right)\right) \rightarrow\left(\frac{x}{2^{2}} \oplus \frac{\sqrt{x}}{2^{2}}\right)$,

IP2. $1=\left(\frac{1}{2^{4}} \oplus\left(\frac{\neg x \bullet \neg x}{2^{2}} \oplus \frac{\sqrt{x} \bullet \sqrt{x}}{2^{2}}\right)\right) \rightarrow\left(\frac{\neg x}{2^{2}} \oplus \frac{\sqrt{x}}{2^{2}}\right)$,

IP3. $1=\left(\frac{1}{2^{4}} \oplus\left(\frac{x \bullet x}{2^{2}} \oplus \frac{\sqrt{\neg x} \bullet \sqrt{\neg x}}{2^{2}}\right)\right) \rightarrow\left(\frac{x}{4} \oplus \frac{\sqrt{\neg x}}{2^{2}}\right)$,

IP4. $1=\left(\frac{1}{2^{4}} \oplus\left(\frac{\neg x \bullet \neg x}{2^{2}} \oplus \frac{\sqrt{\neg x} \bullet \sqrt{\neg x}}{2^{2}}\right)\right) \rightarrow\left(\frac{\neg x}{2^{2}} \oplus \frac{\sqrt{\neg x}}{2^{2}}\right)$,

IP5. $\frac{1}{2^{4}} \oplus \frac{1}{2^{4}}=\frac{1}{2^{3}}$.

where $\frac{1}{2^{n}}$ means the term $\frac{1}{2^{n-1}} \bullet \frac{1}{2}$ and $\frac{t}{2^{n}}$ means the term $t \bullet \frac{1}{2^{n}}(n \geq 2)$. 
Remark 5.2 In the particular case of $D_{[0,1]}$, axioms IP1,..,IP4 are all equivalent. This is due to simple arithmetic properties of the real numbers. In the general case, a $\sqrt{q P M V}$-algebra that satisfies one of these axioms does not necessarily satisfy the others. It is due to this fact that the four equations must be introduce as axioms in the definition of $I P$-algebra.

We denote by $\mathcal{I P}$ the subvariety of $\sqrt{q \mathcal{P M V}}$ given by the $I P$-algebras. Clearly $\mathcal{I P}$-homomorphisms are $\sqrt{q \mathcal{P} \mathcal{M V}}$-homomorphisms. $D_{[0,1]}$ is an $I P$ algebra and constitutes the standard model for $\mathcal{I P}$. Another important examples of $I P$-algebras are the flat algebras.

Remark 5.3 Unfortunately we cannot give a completeness theorem for the $\mathcal{I P}$-equations of the form $t=s$ with respect to $D_{[0,1]}$. In fact, the open problem for the axiomatization of all identities in the language of $\mathcal{P} \mathcal{M V}$ which are valid in the $P M V$-algebra arising from the real interval $[0,1]$ (see $[22,16])$ will appear in $\mathcal{I} \mathcal{P}$. In view of this, we delineate a generalization of the $D_{[0,1]}$ algebra, whose role is analogous to the $P M V$-chains with respect to the equational theory of $\mathcal{P} \mathcal{M V}$.

Let $A$ be a $P M V_{\frac{1}{2^{4}}}$-algebra (see Definition 2.3) and $S_{A}$ be the pair algebra over $A$. We consider the following subsets in $S_{A}$ :

$$
\begin{aligned}
& Q_{1}=\left\{x \in S_{A}: 1=\left(\frac{1}{2^{4}} \oplus\left(\frac{x \bullet x}{2^{2}} \oplus \frac{\sqrt{x} \bullet \sqrt{x}}{2^{2}}\right)\right) \rightarrow\left(\frac{x}{2^{2}} \oplus \frac{\sqrt{x}}{2^{2}}\right)\right\} \\
& Q_{2}=\left\{x \in S_{A}: 1=\left(\frac{1}{2^{4}} \oplus\left(\frac{\neg x \bullet \neg x}{2^{2}} \oplus \frac{\sqrt{x} \bullet \sqrt{x}}{2^{2}}\right)\right) \rightarrow\left(\frac{\neg x}{2^{2}} \oplus \frac{\sqrt{x}}{2^{2}}\right)\right\} \\
& Q_{3}=\left\{x \in S_{A}: 1=\left(\frac{1}{2^{4}} \oplus\left(\frac{x \bullet x}{2^{2}} \oplus \frac{\sqrt{\neg x} \bullet \sqrt{\neg x}}{2^{2}}\right)\right) \rightarrow\left(\frac{x}{2^{2}} \oplus \frac{\sqrt{\neg x}}{2^{2}}\right)\right\} \\
& Q_{4}=\left\{x \in S_{A}: 1=\left(\frac{1}{2^{4}} \oplus\left(\frac{\neg x \bullet \neg x}{2^{2}} \oplus \frac{\sqrt{\neg x} \cdot \sqrt{\neg x}}{2^{2}}\right)\right) \rightarrow\left(\frac{\neg x}{2^{2}} \oplus \frac{\sqrt{\neg x}}{2^{2}}\right)\right\}
\end{aligned}
$$

Then we define

$$
D_{A}=Q 1 \cap Q 2 \cap Q 3 \cap Q 4
$$

Proposition 5.4 Let $S_{A}$ be the pair algebra over the $P M V_{\frac{1}{2^{4}}}$-algebra $A$. Then:

$$
\left\langle D_{A}, \oplus, \bullet, \sqrt{ },\left(0, \frac{1}{2}\right),\left(\frac{1}{2}, \frac{1}{2}\right),\left(1, \frac{1}{2}\right)\right\rangle
$$

is the largest IP-algebra contained in $S_{A}$ as sub- $\sqrt{q P M V}$-algebra. Moreover $\left(a, \frac{1}{2}\right) \mapsto$ a defines a $\mathcal{P} \mathcal{M} \mathcal{V}_{\frac{1}{2^{4}}}$-isomorphic from $\operatorname{Reg}\left(D_{A}\right)$ onto $A$. 
Proof: We first prove that $\operatorname{Reg}\left(S_{A}\right) \subseteq D_{A}$. Let $\left(a, \frac{1}{2}\right) \in \operatorname{Reg}\left(S_{A}\right)$. We have to prove the following four cases:

Case 1: $\left.\left(a, \frac{1}{2}\right) \in Q_{1} \cdot \quad \frac{1}{2^{4}} \oplus\left(\frac{x \bullet x}{2^{2}} \oplus \frac{\sqrt{x} \bullet \sqrt{x}}{2^{2}}\right)\right|_{\left(a, \frac{1}{2}\right)}=\left(\frac{1}{2^{4}}, \frac{1}{2}\right) \oplus\left(\left(\frac{1}{2^{2}}, \frac{1}{2}\right)\right.$ $\left.\left(a, \frac{1}{2}\right) \bullet\left(a, \frac{1}{2}\right)\right) \oplus\left(\left(\frac{1}{2^{2}}, \frac{1}{2}\right) \bullet \sqrt{\left(a, \frac{1}{2}\right)} \bullet \sqrt{\left(a, \frac{1}{2}\right)}\right)=\left(\frac{1}{2^{4}}, \frac{1}{2}\right) \oplus\left(\frac{a \bullet a}{2^{2}}, \frac{1}{2}\right) \oplus\left(\frac{1}{2^{4}}, \frac{1}{2}\right)=$ $\left(\frac{a \bullet a}{2^{2}} \oplus \frac{1}{2^{3}}, \frac{1}{2}\right)$ since $A$ is a $P M V_{\frac{1}{2^{4}}}$-algebra, i.e. $\frac{1}{2^{4}} \oplus \frac{1}{2^{4}}=\frac{1}{2^{3}}$ holds in the first component.

$$
\left.\frac{x}{2^{2}} \oplus \frac{\sqrt{x}}{2^{2}}\right|_{\left(a, \frac{1}{2}\right)}=\left(\left(\frac{1}{2^{2}}, \frac{1}{2}\right) \bullet\left(a, \frac{1}{2}\right)\right) \oplus\left(\left(\frac{1}{2^{2}}, \frac{1}{2}\right) \bullet \sqrt{\left(a, \frac{1}{2}\right)}\right)=\left(\frac{a}{2^{2}} \oplus \frac{1}{2^{3}}, \frac{1}{2}\right) .
$$

Since $\frac{a \bullet a}{2^{2}} \oplus \frac{1}{2^{3}} \leq \frac{a}{2^{2}} \oplus \frac{1}{2^{3}}$ in the $P M V_{\frac{1}{2^{4}}}$-algebra $A,\left.\frac{1}{2^{4}} \oplus\left(\frac{x \bullet x}{2^{2}} \oplus \frac{\sqrt{x} \bullet \sqrt{x}}{2^{2}}\right)\right|_{\left(a, \frac{1}{2}\right)} \leq$ $\left.\frac{x}{2^{2}} \oplus \frac{\sqrt{x}}{2^{2}}\right|_{\left(a, \frac{1}{2}\right)}$. Hence $\left(a, \frac{1}{2}\right) \in Q_{1}$ for each $a \in A$.

Case 2: $\left.\left(a, \frac{1}{2}\right) \in Q_{2} \cdot \frac{1}{2^{4}} \oplus\left(\frac{\neg x \bullet \neg x}{2^{2}} \oplus \frac{\sqrt{x} \bullet \sqrt{x}}{2^{2}}\right)\right|_{\left(a, \frac{1}{2}\right)}=\left(\frac{1}{2^{4}}, \frac{1}{2}\right) \oplus\left(\left(\frac{1}{2^{2}}, \frac{1}{2}\right) \bullet\right.$ $\left.\left(\neg a, \frac{1}{2}\right) \bullet\left(\neg a, \frac{1}{2}\right)\right) \oplus\left(\left(\frac{1}{2^{2}}, \frac{1}{2}\right) \bullet \sqrt{\left(a, \frac{1}{2}\right)} \bullet \sqrt{\left(a, \frac{1}{2}\right)}\right)=\left(\frac{1}{2^{4}}, \frac{1}{2}\right) \oplus\left(\frac{\neg a \bullet \neg a}{2^{2}}, \frac{1}{2}\right) \oplus$ $\left(\frac{1}{2^{4}}, \frac{1}{2}\right)=\left(\frac{\neg a \bullet \neg a}{2^{2}} \oplus \frac{1}{2^{3}}, \frac{1}{2}\right)$.

$$
\left.\frac{x}{2^{2}} \oplus \frac{\sqrt{x}}{2^{2}}\right|_{\left(\neg a, \frac{1}{2}\right)}=\left(\left(\frac{1}{2^{2}}, \frac{1}{2}\right) \bullet\left(\neg a, \frac{1}{2}\right)\right) \oplus\left(\left(\frac{1}{2^{2}}, \frac{1}{2}\right) \bullet \sqrt{\left(a, \frac{1}{2}\right)}\right)=\left(\frac{\neg a}{2^{2}} \oplus \frac{1}{2^{3}}, \frac{1}{2}\right) .
$$

Since $\frac{\neg a \bullet \neg a}{2^{2}} \oplus \frac{1}{2^{3}} \leq \frac{\neg a}{2^{2}} \oplus \frac{1}{2^{3}}$ in the $P M V_{\frac{1}{2^{4}}}$-algebra $A, \frac{1}{2^{4}} \oplus\left(\frac{\neg x \bullet \neg x}{2^{2}} \oplus\right.$ $\left.\frac{\sqrt{x} \cdot \sqrt{x}}{2^{2}}\right)\left.\right|_{\left(a, \frac{1}{2}\right)} \leq\left.\frac{7 x}{2^{2}} \oplus \frac{\sqrt{x}}{2^{2}}\right|_{\left(a, \frac{1}{2}\right)}$. Hence $\left(a, \frac{1}{2}\right) \in Q_{2}$ for each $a \in A$.

Case 3: $\left(a, \frac{1}{2}\right) \in Q_{3}:\left.\frac{1}{2^{4}} \oplus\left(\frac{x \bullet x}{2^{2}} \oplus \frac{\sqrt{\neg x} \bullet \sqrt{\neg x}}{2^{2}}\right)\right|_{\left(a, \frac{1}{2}\right)}=\left(\frac{1}{2^{4}}, \frac{1}{2}\right) \oplus\left(\left(\frac{1}{2^{2}}, \frac{1}{2}\right)\right.$ $\left.\left(a, \frac{1}{2}\right) \bullet\left(a, \frac{1}{2}\right)\right) \oplus\left(\left(\frac{1}{2^{2}}, \frac{1}{2}\right) \bullet \sqrt{\neg\left(a, \frac{1}{2}\right)} \bullet \sqrt{\neg\left(a, \frac{1}{2}\right)}\right)=\left(\frac{1}{2^{4}}, \frac{1}{2}\right) \oplus\left(\frac{a \bullet a}{2^{2}}, \frac{1}{2}\right) \oplus\left(\frac{1}{2^{4}}, \frac{1}{2}\right)=$ $\left(\frac{a \bullet a}{2^{2}} \oplus \frac{1}{2^{3}}, \frac{1}{2}\right)$ since $A$ is a $P M V_{\frac{1}{2^{4}}}$-algebra, i.e. $\frac{1}{2^{4}} \oplus \frac{1}{2^{4}}=\frac{1}{2^{3}}$ holds in the first component.

$$
\left.\frac{x}{2^{2}} \oplus \frac{\sqrt{\neg x}}{2^{2}}\right|_{\left(a, \frac{1}{2}\right)}=\left(\left(\frac{1}{2^{2}}, \frac{1}{2}\right) \bullet\left(a, \frac{1}{2}\right)\right) \oplus\left(\left(\frac{1}{2^{2}}, \frac{1}{2}\right) \bullet \sqrt{\neg\left(a, \frac{1}{2}\right)}\right)=\left(\frac{a}{2^{2}} \oplus \frac{1}{2^{3}}, \frac{1}{2}\right) .
$$

Since $\frac{a \bullet a}{2^{2}} \oplus \frac{1}{2^{3}} \leq \frac{a}{2^{2}} \oplus \frac{1}{2^{3}}$ in the $P M V_{\frac{1}{2^{4}}}$-algebra $A,\left.\frac{1}{2^{4}} \oplus\left(\frac{x \bullet x}{2^{2}} \oplus \frac{\sqrt{\neg x} \bullet \sqrt{\neg x}}{2^{2}}\right)\right|_{\left(a, \frac{1}{2}\right)} \leq$ $\left.\frac{x}{2^{2}} \oplus \frac{\sqrt{\neg x}}{2^{2}}\right|_{\left(a, \frac{1}{2}\right)}$. Hence $\left(a, \frac{1}{2}\right) \in Q_{3}$ for each $a \in A$.

Case 4: $\left.\left(a, \frac{1}{2}\right) \in Q_{4} \cdot \frac{1}{2^{4}} \oplus\left(\frac{\neg x \bullet \neg x}{2^{2}} \oplus \frac{\sqrt{\neg x} \bullet \sqrt{\neg x}}{2^{2}}\right)\right|_{\left(a, \frac{1}{2}\right)}=\left(\frac{1}{2^{4}}, \frac{1}{2}\right) \oplus\left(\left(\frac{1}{2^{2}}, \frac{1}{2}\right) \bullet\right.$ $\left.\left(\neg a, \frac{1}{2}\right) \bullet\left(\neg a, \frac{1}{2}\right)\right) \oplus\left(\left(\frac{1}{2^{2}}, \frac{1}{2}\right) \bullet \sqrt{\neg\left(a, \frac{1}{2}\right)} \bullet \sqrt{\neg\left(a, \frac{1}{2}\right)}\right)=\left(\frac{1}{2^{4}}, \frac{1}{2}\right) \oplus\left(\frac{\neg a \bullet \neg a}{2^{2}}, \frac{1}{2}\right) \oplus$ $\left(\frac{1}{2^{4}}, \frac{1}{2}\right)=\left(\frac{\neg a \bullet \neg a}{2^{2}} \oplus \frac{1}{2^{3}}, \frac{1}{2}\right)$.

$$
\left.\frac{\neg x}{2^{2}} \oplus \frac{\sqrt{\neg x}}{2^{2}}\right|_{\left(\neg a, \frac{1}{2}\right)}=\left(\left(\frac{1}{2^{2}}, \frac{1}{2}\right) \bullet\left(\neg a, \frac{1}{2}\right)\right) \oplus\left(\left(\frac{1}{2^{2}}, \frac{1}{2}\right) \bullet \sqrt{\neg\left(a, \frac{1}{2}\right)}\right)=\left(\frac{\neg a}{2^{2}} \oplus \frac{1}{2^{3}}, \frac{1}{2}\right) .
$$


Since $\frac{\neg a \bullet \neg a}{2^{2}} \oplus \frac{1}{2^{3}} \leq \frac{\neg a}{2^{2}} \oplus \frac{1}{2^{3}}$ in the $P M V_{\frac{1}{2^{4}}}$-algebra $A, \frac{1}{2^{4}} \oplus\left(\frac{\neg x \bullet \neg x}{2^{2}} \oplus\right.$ $\left.\frac{\sqrt{\neg x} \bullet \sqrt{\neg x}}{2^{2}}\right)\left.\right|_{\left(a, \frac{1}{2}\right)} \leq\left.\frac{\neg x}{2^{2}} \oplus \frac{\sqrt{\neg x}}{2^{2}}\right|_{\left(a, \frac{1}{2}\right)}$. Hence $\left(a, \frac{1}{2}\right) \in Q_{4}$ for each $a \in A$.

Hence $\left(a, \frac{1}{2}\right) \in D_{A}$ for each $a \in A$ and $\operatorname{Reg}\left(S_{A}\right) \subseteq D_{A}$.

Now we prove that $D_{A}$ defines a sub $\sqrt{q P M V}$-algebra of $S_{A}$.

- $D_{A}$ is closed by $\star \in\{\oplus, \bullet\}$. Let $\left(x_{1}, y_{1}\right),\left(x_{2}, y_{2}\right)$ in $D_{A}$ and $\star \epsilon$ $\{\oplus, \bullet\}$. By the precedent argument, $\left(x_{1}, y_{1}\right) \star\left(x_{2}, y_{2}\right)=\left(x_{1} \star x_{2}, \frac{1}{2}\right) \in$ $\operatorname{Reg}\left(S_{A}\right) \subseteq D_{A}$. Hence, $D_{A}$ is closed by $\oplus$ and $\bullet$.

- $D_{A}$ is closed by $\sqrt{ }$. Let $t \in D_{A}$. We have to prove the following four cases:

Case 1: $\left.\sqrt{t} \in Q_{1} \cdot\left(\frac{1}{2^{4}} \oplus\left(\frac{x \bullet x}{2^{2}} \oplus \frac{\sqrt{x} \bullet \sqrt{x}}{2^{2}}\right)\right) \rightarrow\left(\frac{x}{2^{2}} \oplus \frac{\sqrt{x}}{2^{2}}\right)\right|_{\sqrt{t}}=\left(\frac{1}{2^{4}} \oplus\left(\frac{\sqrt{t} \bullet \sqrt{t}}{2^{2}} \oplus\right.\right.$ $\left.\left.\frac{\sqrt{\sqrt{t}} \cdot \sqrt{\sqrt{t}}}{2^{2}}\right)\right) \rightarrow\left(\frac{\sqrt{t}}{2^{2}} \oplus \frac{\sqrt{\sqrt{t}}}{2^{2}}\right)=\left(\frac{1}{2^{4}} \oplus\left(\frac{\sqrt{t} \bullet \sqrt{t}}{2^{2}} \oplus \frac{\neg t \bullet \neg t}{2^{2}}\right)\right) \rightarrow\left(\frac{\sqrt{t}}{2^{2}} \oplus \frac{\neg t}{2^{2}}\right)=1$ since $t \in Q_{2}$. Hence, $\sqrt{t} \in Q_{1}$.

Case 2: $\sqrt{t} \in Q_{2} .\left.\quad\left(\frac{1}{2^{4}} \oplus\left(\frac{\neg x \bullet \neg x}{2^{2}} \oplus \frac{\sqrt{x} \bullet \sqrt{x}}{2^{2}}\right)\right) \rightarrow\left(\frac{\neg x}{2^{2}} \oplus \frac{\sqrt{x}}{2^{2}}\right)\right|_{\sqrt{t}}=\left(\frac{1}{2^{4}} \oplus\right.$ $\left.\left(\frac{\sqrt{\neg t} \bullet \sqrt{\neg t}}{2^{2}} \oplus \frac{\sqrt{\sqrt{t} \bullet \sqrt{\sqrt{t}}}}{2^{2}}\right)\right) \rightarrow\left(\frac{\sqrt{\neg t}}{2^{2}} \oplus \frac{\sqrt{\sqrt{t}}}{2^{2}}\right)=\left(\frac{1}{2^{4}} \oplus\left(\frac{\sqrt{\neg t} \bullet \sqrt{\neg t}}{2^{2}} \oplus \frac{\neg t \bullet \neg t}{2^{2}}\right)\right) \rightarrow$ $\left(\frac{\sqrt{\neg t}}{2^{2}} \oplus \frac{\neg t}{2^{2}}\right)=1$ since $t \in Q_{4}$.

Case 3: $\sqrt{t} \in Q_{3} .\left.\quad\left(\frac{1}{2^{4}} \oplus\left(\frac{x \bullet x}{2^{2}} \oplus \frac{\sqrt{\neg x} \bullet \sqrt{\neg x}}{2^{2}}\right)\right) \rightarrow\left(\frac{x}{2^{2}} \oplus \frac{\sqrt{\neg x}}{2^{2}}\right)\right|_{\sqrt{t}}=\left(\frac{1}{2^{4}} \oplus\right.$

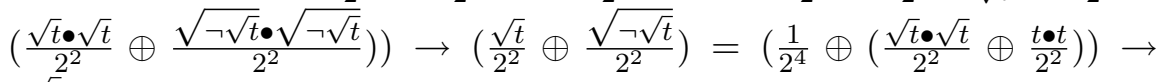
$\left(\frac{\sqrt{t}}{2^{2}} \oplus \frac{t}{2^{2}}\right)=1$ since $t \in Q_{1}$. Hence, $\sqrt{t} \in Q_{3}$.

Case 4: $\sqrt{t} \in Q_{4} .\left.\quad\left(\frac{1}{2^{4}} \oplus\left(\frac{\neg x \bullet \neg x}{2^{2}} \oplus \frac{\sqrt{\neg x} \bullet \sqrt{\neg x}}{2^{2}}\right)\right) \rightarrow\left(\frac{\neg x}{2^{2}} \oplus \frac{\sqrt{\neg x}}{2^{2}}\right)\right|_{\sqrt{t}}=\left(\frac{1}{2^{4}} \oplus\right.$

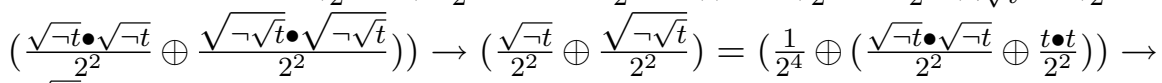
$\left(\frac{\sqrt{-t}}{2^{2}} \oplus \frac{t}{2^{2}}\right)=1$ since $t \in Q_{3}$. Hence, $\sqrt{t} \in Q_{4}$.

Hence $\sqrt{t} \in D_{A}$ and $D_{A}$ is closed by $\sqrt{ }$.

Thus $D_{A}$ is a sub- $\sqrt{q P M V}$-algebra of $S_{A}$. Since $\operatorname{Reg}\left(S_{A}\right) \subseteq D_{A}, \operatorname{Reg}\left(D_{A}\right)$ is $\mathcal{P M V}{\frac{1}{2^{4}}}$-isomorphic to $A$. By definition of $D_{A}$ it is immediate that $D_{A}$ is an $I P$-algebra. Let $B$ be an $I P$-algebra such that $B$ is a sub- $\sqrt{q P M V}$-algebra of $S_{A}$. If $a \in B$, then $a$ satisfies IP1,... IP4. Then $s \in D_{A}$ and $B \subseteq D_{A}$. Hence $D_{A}$ is the largest $I P$-algebra contained in $S_{A}$ as sub- $\sqrt{q P M V}$-algebra. 
Theorem 5.5 Let $\mathcal{S}^{\circ}$ be the sub-class of IP-algebras $D_{A}$ where $A$ is a $P M V_{\frac{1}{2^{4}}}$-chain. Then:

$$
\mathcal{I P}=\mathcal{V}\left(\mathcal{S}^{\circ}\right)
$$

Proof: $\quad$ We shall prove that $\mathcal{I P} \models t=s$ iff $\mathcal{S}^{\circ} \models t=s$. As regards the non-trivial direction, assume that $\mathcal{S}^{\circ} \models t=s$. Suppose that there exists an $I P$-algebra $A$ such that $A \not \forall t=s$. By Proposition 4.10 , there exists a $\sqrt{q \mathcal{P M V}}$-embedding $f: A \rightarrow F l(A) \times S_{\operatorname{Reg}(A)}$. Thus the image $f(A)$ is an

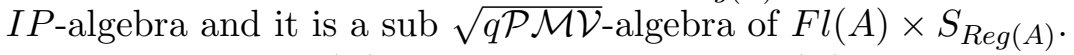

We prove that $f(A)$ is a sub $I P$-algebra of $F l(A) \times D_{R e g(A)}$. Let $\left(a_{1}, a_{2}\right) \in$ $f(A)$. Then there exists $a \in A$ such that $f(a)=\left(a_{1}, a_{2}\right)$. Since $a$ satisfies IP $1, \ldots, \mathrm{IP} 4, f(a)$ also satisfies these equations. It implies that $a_{2}$ satisfies IP1,..,IP4 and by Proposition 5.4, $a_{2} \in D_{\operatorname{Reg}(A)}$. This proves that $f(a)=$ $\left(a_{1}, a_{2}\right) \in F l(A) \times D_{\operatorname{Reg}(A)}$ and then, $f(A)$ is a sub $I P$-algebra of $F l(A) \times$ $D_{\operatorname{Reg}(A)}$. Consequently $F l(A) \times D_{\operatorname{Reg}(A)} \not \neq t=s$ and, by Proposition 4.9-5, $D_{\operatorname{Reg}(A)} \not \forall t=s$.

By Proposition 2.2, consider a subdirect representation $\beta: \operatorname{Reg}(A) \hookrightarrow$ $\prod_{i \in I} A_{i}$ such that $\left(A_{i}\right)_{i \in I}$ is a family of $P M V_{\frac{1}{2^{4}}}$-chains. For each $i \in I$, let $p_{i}$ be the $i$ th-projection in $A_{i}$ and consider the following function:

$$
\beta_{i}: D_{R e g(A)} \rightarrow S_{A_{i}} \quad \text { s.t. } \quad(x, y) \mapsto \beta_{i}((x, y))=\left(p_{i} \beta(x), p_{i} \beta(y)\right)_{i \in I}
$$

Following the same argument used in the proof of Theorem 4.11 we can prove

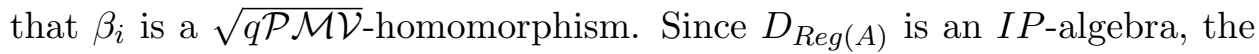
image $\beta_{i}\left(D_{\operatorname{Reg}(A)}\right)$ is an $I P$-algebra. Then, by Proposition 5.4, $\beta_{i}\left(D_{\operatorname{Reg}(A)}\right)$ is a sub $I P$-algebra of $D_{A_{i}}$. In other words, we can see $\beta_{i}$ as a $\sqrt{q \mathcal{P} \mathcal{M} \mathcal{V}}$ homomorphism $\beta_{i}: D_{\operatorname{Reg}(A)} \rightarrow D_{A_{i}}$ for each $i \in I$. Now we define the function

$$
\beta^{*}: D_{\operatorname{Reg}(A)} \rightarrow \Pi_{i \in I} D_{A_{i}} \quad \text { s.t. } \quad(x, y) \mapsto \beta^{*}((x, y))=\left(\beta_{i}(x, y)\right)_{i \in I}
$$

$\beta^{*}$ is injective since $\beta$ is injective. Moreover $\beta^{*}$ is a $\sqrt{q \mathcal{P} \mathcal{M V}}$-homomorphism since $\beta_{i}$ is a $\sqrt{q \mathcal{P} \mathcal{M V}}$-homomorphism for each $i \in I$.

Thus $D_{\operatorname{Reg}(A)} \not \neq t=s$ implies that there exists $m \in I$ such that $D_{A_{i}} \not$ $t=s$ which is a contradiction since $D_{A_{i}} \in \mathcal{S}^{\circ}$. Hence $\mathcal{I P} \models t=s$.

\section{Probabilistic consequence}

An usual problem treated in digital techniques is the following: if $T$ is a set of Boolean circuits and $t$ is a Boolean circuit, we want to know if a 
determinate state of the outputs of the circuits of $T$, represented in a string of bits 0,1 , forces a determinate state of the output of $t$ given by a bit, either 0 or 1 . As a general rule, this problem can be solved through effective procedures solving the particular case in which a set of circuits $T$ with all outputs in state 1 forces the state 1 in the output of a circuit $t$.

One may naturally extend this problem by considering circuits made from assemblies of quantum gates of the $\mathbb{I P}$-system called $\mathbb{I P}$-circuits. In fact: let $T$ be a set of $\mathbb{I P}$-circuits and $t$ be an $\mathbb{I P}$-circuit. Suppose that the output of the circuits of $T$ are labeled with density operators $\left(\sigma_{i}\right)_{i}$ such that $p\left(\sigma_{i}\right)=1$ for each $i$. We want to know whether from the above, necessarily follows an output of $t$ labeled with a density operator $\sigma$ such that $p(\sigma)=1$. Since each $\mathbb{I P}$-circuit can be related to a $\sqrt{q \mathcal{P \mathcal { M V }}}$-term we can define a relation of consequence based on the preservation of probability values 1 .

Definition 6.1 Let $t \in \operatorname{Term}_{\sqrt{q \mathcal{P M N}}}$ and $T \subseteq \operatorname{Term}_{\sqrt{q \mathcal{P \mathcal { M }} V}}$. We say that $t$ is a probabilistic consequence of $T$ in $\mathcal{D}\left(\mathbb{C}^{2}\right)$ (noted $\left.T \models_{\mathcal{D}\left(\mathbb{C}^{2}\right)}^{\text {Prob }} t\right)$ iff for each valuation $e: \operatorname{Term}_{\sqrt{q \mathcal{P M V}}} \rightarrow \mathcal{D}\left(\mathbb{C}^{2}\right), p(e(t))=1$ whenever $p(e(s))=1$ for each $s \in T$.

Taking into account Proposition 3.4 and Remark 3.5, for each $\sqrt{q \mathcal{P M \mathcal { V }}}$ term $t$ and for each valuation $e: \operatorname{Term}_{\sqrt{q \mathcal{P M \mathcal { N }}}} \rightarrow \mathcal{D}\left(\mathbb{C}^{2}\right)$, the probability valued $p(e(t))$ can be identifies with $e(t \oplus 0)$. Thus we can establish the following result:

Proposition 6.2 Let $t \in$ Term $_{\sqrt{q \mathcal{P M V}}}$ and $T \subseteq$ Term $_{\sqrt{q \mathcal{P M V}}}$. Then the following conditions are equivalent:

1. $T \models \underset{\mathcal{D}\left(\mathbb{C}^{2}\right)}{\text { rob }} t$.

2. For each valuation $e: \operatorname{Term}_{\sqrt{q \mathcal{P M V}}} \rightarrow \mathcal{D}\left(\mathbb{C}^{2}\right)$, $e(t \oplus 0)=P_{1}$ whenever $e(s \oplus 0)=P_{1}$ for each $s \in T$.

The equivalence given in Proposition 6.2 allows to extend, in a natural way, the concepts of probability assignment and probabilistic consequence with respect to each $I P$-algebra.

Definition 6.3 Let $A$ be an $I P$-algebra, e: $\operatorname{Term}_{\sqrt{q \mathcal{P M V}}} \rightarrow A$ be a valuation and $t \in$ Term $_{\sqrt{q \mathcal{P M V}}}$. Then we define the generalized probability value associated to $e$ as $e_{p}(t)=e(t \oplus 0)$. 
We introduce the following notation: Let $T \subseteq$ Term $_{\sqrt{q \mathcal{P M} \mathcal{V}}}$ and $e$ : $\operatorname{Term}_{\sqrt{q \mathcal{P M V}}} \rightarrow A$ be a valuation. Then $e_{p}(T)=1$ means that for each $s \in T, e_{p}(s)=1$.

Definition 6.4 Let $t \in \operatorname{Term}_{\sqrt{q \mathcal{P M \mathcal { N }}}}, T \subseteq \operatorname{Term}_{\sqrt{q \mathcal{P M \mathcal { V }}}}$ and $A$ be an $I P$ algebra. We say that $t$ is a probabilistic consequence of $T$ in $A$ iff for each valuation $e: \operatorname{Term}_{\sqrt{q \mathcal{P \mathcal { M }}}} \rightarrow A$, if $e_{p}(T)=1$ then $e_{p}(t)=1$.

We preserve the notation $T \models_{A}^{P r o b} t$ for the probabilistic consequence in $A$. In particular $T=_{\mathcal{I} P}^{\text {Prob }} t$ means that $T \models_{A}^{\text {Prob }} t$ for each $A \in \mathcal{I P}$.

A $\sqrt{q \mathcal{P} \mathcal{M V}}$-term $t$ is said to be a tautology iff for each $A \in \mathcal{I P}$ and for each $e:$ Term $_{\sqrt{q \mathcal{P M V}}} \rightarrow A, e_{p}(t)=1$. Note that $t$ is a tautology iff $\emptyset \models{ }_{\mathcal{I} \mathcal{P}}^{\text {rob }} t$. Thus we use the notation $\models_{\mathcal{I} \mathcal{P}}^{\text {rob }} t$ in the case in which $t$ is a tautology.

Proposition 6.5 Let $D_{A} \in \mathcal{S}^{\circ}$ where $A$ is a PMV $\frac{1}{2^{4}}$-algebra. If e, $e^{\prime}$ are two valuation over $D_{A}$ such that for each atomic term $\alpha, e_{p}(\alpha)=e_{p}^{\prime}(\alpha)$ and $e_{p}(\sqrt{\alpha})=e_{p}^{\prime}(\sqrt{\alpha})$ then, $e=e^{\prime}$.

Proof: By definition of valuation, we have to see that $e$ and $e^{\prime}$ coincide over atomic terms. Let $\alpha$ be an atomic term. Suppose that $e(\alpha)=(a, b)$ and $e^{\prime}(\alpha)=\left(a^{\prime}, b^{\prime}\right)$. On the one hand, $\left(a, \frac{1}{2}\right)=(a, b) \oplus 0=e_{p}(\alpha)=e_{p}^{\prime}(\alpha)=$ $\left(a^{\prime}, b^{\prime}\right) \oplus 0=\left(a^{\prime}, \frac{1}{2}\right)$ and then, $a=a^{\prime}$. On the other hand, $\left(b^{\prime}, \frac{1}{2}\right)=\left(b^{\prime}, \neg a^{\prime}\right) \oplus$ $0=e_{p}(\sqrt{\alpha})=e_{p}^{\prime}(\sqrt{\alpha})=\left(b^{\prime}, \neg a^{\prime}\right) \oplus 0=\left(b^{\prime}, \frac{1}{2}\right)$ and then, $b=b^{\prime}$. Hence $e(\alpha)=e^{\prime}(\alpha)$.

\section{$7 \quad$ Hilbert system for the probabilistic consequence}

Let $t \in \operatorname{Term}_{\sqrt{q \mathcal{P M \mathcal { V }}}}$ and $T \subseteq \operatorname{Term}_{\sqrt{q \mathcal{P \mathcal { M V }}}}$. One may naturally consider the following decision problem: does there exist an effective procedure deciding whether $T=_{\mathcal{I} \mathcal{P}}^{\text {rob }} t$ ? In this section we shall reformulate this problem in purely logical terms within a Hilbert-style axiomatization $(\mathcal{L I} \mathcal{P})$ for the probabilistic consequence.

Definition 7.1 Consider the absolutely free algebra Term $_{\sqrt{q \mathcal{P M V}}}$ taking into account the following syntactic abbreviations:

$\neg t$ is a syntactic abbreviation for $\sqrt{\sqrt{t}}$, 
$t_{1} \odot t_{2}$ is a syntactic abbreviation for $\neg\left(\neg t_{1} \oplus \neg t_{2}\right)$,

$t_{1} \rightarrow t_{2}$ is a syntactic abbreviation for $\neg t_{1} \oplus t_{2}$,

$t_{1} \leftrightarrow t_{2}$ is a syntactic abbreviation for $\left(t_{1} \rightarrow t_{2}\right) \odot\left(t_{2} \rightarrow t_{1}\right)$,

$\frac{1}{2^{n}}$ is a syntactic abbreviation for $\frac{1}{2^{n-1}} \bullet \frac{1}{2}(n \geq 2)$,

$\frac{t}{2^{n}}$ is a syntactic abbreviation for $t \bullet \frac{1}{2^{n}}(n \geq 2)$.

An axiom of the Hilbert-style calculus $\mathcal{L I} \mathcal{P}$ is a $\sqrt{q \mathcal{P} \mathcal{M V}}$-term that can be written in any one of the following ways, where $\alpha, \beta$ and $\gamma$ denote arbitrary terms in Term $_{\sqrt{q \mathcal{P} \mathcal{M V}}}$ :

\section{Eukasiewicz axioms:}

$\mathrm{W} 1 \alpha \rightarrow(\beta \rightarrow \alpha)$,

$\mathrm{W} 2(\alpha \rightarrow \beta) \rightarrow((\beta \rightarrow \gamma) \rightarrow(\alpha \rightarrow \gamma))$,

$\mathrm{W} 3(\neg \alpha \rightarrow \neg \beta) \rightarrow(\beta \rightarrow \alpha)$,

$\mathrm{W} 4((\alpha \rightarrow \beta) \rightarrow \beta) \rightarrow((\beta \rightarrow \alpha) \rightarrow \alpha)$,

\section{Constant axioms:}

C1 1,

$\mathrm{C} 2 \neg 0 \leftrightarrow 1$,

C3 $\neg \frac{1}{2} \leftrightarrow \frac{1}{2}$,

C4 $\left(\frac{1}{2^{4}} \oplus \frac{1}{2^{4}}\right) \leftrightarrow \frac{1}{2^{3}}$,

Product axioms:

$\mathrm{P} 1(\alpha \bullet \beta) \rightarrow(\beta \bullet \alpha)$,

$\mathrm{P} 2(1 \bullet \alpha) \leftrightarrow \alpha$,

P3 $(\alpha \bullet \beta) \rightarrow \beta$,

$\mathrm{P} 4((\alpha \bullet \beta) \bullet \gamma) \leftrightarrow(\alpha \bullet(\beta \bullet \gamma))$,

P5 $(\alpha \bullet(\beta \odot \neg \gamma)) \leftrightarrow((\alpha \bullet \beta) \odot \neg(\alpha \bullet \gamma))$, 
Sqrt axioms:

$$
\begin{aligned}
& \text { sQ1 } \sqrt{\neg \alpha} \leftrightarrow \neg \sqrt{\alpha}, \\
& \text { sQ2 } \sqrt{\alpha \star \beta} \leftrightarrow \frac{1}{2} \quad \text { where } \star \in\{\oplus, \bullet\}, \\
& \text { sQ3 } \sqrt{c} \leftrightarrow \frac{1}{2} \quad \text { where } c \in\left\{0, \frac{1}{2}, 1\right\}, \\
& \text { sQ4 }\left(\frac{1}{2^{4}} \oplus\left(\frac{\alpha \bullet \alpha}{2^{2}} \oplus \frac{\sqrt{\alpha} \cdot \sqrt{\alpha}}{2^{2}}\right)\right) \rightarrow\left(\frac{\alpha}{2^{2}} \oplus \frac{\sqrt{\alpha}}{2^{2}}\right), \\
& \text { sQ5 }\left(\frac{1}{2^{4}} \oplus\left(\frac{\neg \alpha \bullet \neg \alpha}{2^{2}} \oplus \frac{\sqrt{\alpha} \bullet \sqrt{\alpha}}{2^{2}}\right)\right) \rightarrow\left(\frac{\neg \alpha}{2^{2}} \oplus \frac{\sqrt{\alpha}}{2^{2}}\right), \\
& \text { sQ6 }\left(\frac{1}{2^{4}} \oplus\left(\frac{\alpha \bullet \alpha}{2^{2}} \oplus \frac{\sqrt{\neg \alpha} \cdot \sqrt{\neg \alpha}}{2^{2}}\right)\right) \rightarrow\left(\frac{\alpha}{2^{2}} \oplus \frac{\sqrt{\neg \alpha}}{2^{2}}\right), \\
& \text { sQ7 }\left(\frac{1}{2^{4}} \oplus\left(\frac{\neg \alpha \bullet \neg \alpha}{2^{2}} \oplus \frac{\sqrt{\neg \alpha} \cdot \sqrt{\neg \alpha}}{2^{2}}\right)\right) \rightarrow\left(\frac{\neg \alpha}{2^{2}} \oplus \frac{\sqrt{\neg \alpha}}{2^{2}}\right) .
\end{aligned}
$$

The deduction rule of $\mathcal{L I P}$ is modus ponens

$$
\{\alpha, \alpha \rightarrow \beta\} \vdash_{\mathcal{L I P}} \beta \quad(M P)
$$

Note that, axioms $\mathrm{W} 1 \ldots \mathrm{W} 4, \mathrm{C} 1, \mathrm{C} 2$ and MP define the same propositional system as the infinite valued Łukasiewicz calculus [4, §4]. By adding $\mathrm{C} 3 \ldots \mathrm{C} 4$ and P1...P5, the propositional system associated to the product Eukasiewikz logic [14, 22] with fix point of the negation [15] is obtained. sQ1, sQ2 and sQ3 axiomatize the basic properties of the operation $\sqrt{ } \cdot$ Finally, sQ4...sQ7 allow to introduce in the calculus the property that characterizes the subvariety of $I P$-algebras with respect to the variety $\sqrt{q \mathcal{P M \mathcal { V }}}$, as was already mentioned at the beginning of Section 5 and in Remark 5.2.

A theory is any set $T \subseteq T e r m_{\sqrt{q \mathcal{P M V}}}$. A proof from $T$ is a sequence of terms $\alpha_{1}, \ldots, \alpha_{n}$ such that each member is either an axiom or a member of $T$ or follows from preceding members of the sequence by modus ponens. The notation $T \vdash_{\mathcal{L I P}} \alpha$ (to be read ' $\alpha$ is provable form $T$ ') means that $\alpha$ is the last term of a proof from $T$. Thus the Hilbert-style calculus $\mathcal{L I P}$ is given by

$$
\mathcal{L I P}=\left\langle\operatorname{Term}_{\sqrt{q \mathcal{P} \mathcal{M V}}}, \vdash_{\mathcal{L I P}}\right\rangle
$$

Let $T$ be a theory. If $T=\emptyset$ we use the notation $\vdash_{\mathcal{L I P}} \alpha$ and we say that $\alpha$ is a theorem of $\mathcal{L I P} . T$ is inconsistent iff $T \vdash_{\mathcal{L I P}} \alpha$ for each $\alpha \in$ $\operatorname{Term}_{\sqrt{q \mathcal{P} \mathcal{M V}}}$; otherwise it is consistent. 
Lemma 7.2 Let $\alpha, \beta \in$ Term $_{\sqrt{q \mathcal{P M V}}}$ and $T \subseteq$ Term $_{\sqrt{q \mathcal{P M V}}}$. Then the following items may be proved using only W1...W4, C1, C2, P1..P5 and $M P$.
1. $\vdash_{\mathcal{L I P}} \alpha \rightarrow \alpha$,
2. $T \vdash_{\mathcal{L I P}} \alpha \odot \beta \quad$ iff $\quad T \vdash_{\mathcal{L I P}} \alpha$ and $T \vdash_{\mathcal{L I P}} \beta$,
3. $T \vdash_{\mathcal{L I P}} \alpha \leftrightarrow \beta \quad$ iff $\quad T \vdash_{\mathcal{L I P}} \alpha \rightarrow \beta$ and $T \vdash_{\mathcal{L I P}} \beta \rightarrow \alpha$,
4. $T \vdash_{\mathcal{L I P}} \alpha \rightarrow \beta$ and $T \vdash_{\mathcal{L I P}} \beta \rightarrow \gamma \quad$ then $\quad T \vdash_{\mathcal{L I P}} \alpha \rightarrow \gamma$,
5. $\vdash_{\mathcal{L I P}} \neg \neg \alpha \rightarrow \alpha$,
6. $\vdash_{\mathcal{L I P}}(\alpha \rightarrow \beta) \rightarrow(\neg \beta \rightarrow \neg \alpha)$,
7. $\vdash_{\mathcal{L I P}}(\alpha \rightarrow \beta) \rightarrow((\alpha \oplus \gamma) \rightarrow(\beta \oplus \gamma))$,
8. $\vdash_{\mathcal{L I P}}((\alpha \leftrightarrow \beta) \odot(\beta \leftrightarrow \gamma)) \rightarrow(\alpha \leftrightarrow \gamma)$,
9. $\vdash_{\mathcal{L I P}}(\alpha \leftrightarrow \beta) \rightarrow((\alpha \rightarrow \gamma) \leftrightarrow(\beta \rightarrow \gamma))$,
10. $\vdash_{\mathcal{L I P}}(\alpha \leftrightarrow \beta) \rightarrow((\gamma \rightarrow \alpha) \leftrightarrow(\gamma \rightarrow \beta))$,
11. $\vdash_{\mathcal{L I P}}(\alpha \rightarrow \beta) \rightarrow((\gamma \bullet \alpha) \rightarrow(\gamma \bullet \beta))$.

Proof: Items $1 \ldots 10$ follow by observing that this result are theorems and metatheorems in the infinite valued Lukasiewicz calculus (see [13]). We prove item 11:

(1) $\vdash_{\mathcal{L I P}} \gamma \bullet(\alpha \odot \neg \beta) \rightarrow((\alpha \odot \neg \beta))$

by Ax P3

$(2) \vdash_{\mathcal{L I P}}((\gamma \bullet \alpha) \odot \neg(\gamma \bullet \beta)) \rightarrow \gamma \bullet(\alpha \odot \neg \beta)$

by Ax P5

$(3) \vdash_{\mathcal{L I P}}((\gamma \bullet \alpha) \odot \neg(\gamma \bullet \beta)) \rightarrow(\alpha \odot \neg \beta)$

by $1,2, \mathrm{Ax} \mathrm{W} 2$

(4) $\vdash_{\mathcal{L I P}}(((\gamma \bullet \alpha) \odot \neg(\gamma \bullet \beta)) \rightarrow(\alpha \odot \neg \beta)) \rightarrow$

$$
(\neg(\alpha \odot \neg \beta) \rightarrow \neg((\gamma \bullet \alpha) \odot \neg(\gamma \bullet \beta))) \quad \text { by Ax W3 }
$$

$(5) \vdash_{\mathcal{L I P}} \neg(\alpha \odot \neg \beta) \rightarrow \neg((\gamma \bullet \alpha) \odot \neg(\gamma \bullet \beta))$

by MP 3,4

(6) $\vdash_{\mathcal{L I P}}(\alpha \rightarrow \beta) \rightarrow \neg(\alpha \odot \neg \beta)$

by $\operatorname{def} \odot$, item 1

$(7) \vdash_{\mathcal{L I P}}(\alpha \rightarrow \beta) \rightarrow \neg((\gamma \bullet \alpha) \odot \neg(\gamma \bullet \beta))$

by $5,6, \mathrm{Ax} \mathrm{W} 2$ 
$(8) \vdash_{\mathcal{L I P}} \neg((\gamma \bullet \alpha) \odot \neg(\gamma \bullet \beta)) \rightarrow((\gamma \bullet \alpha) \rightarrow(\gamma \bullet \beta)) \quad$ by def $\odot$, item 1

$(9) \vdash_{\mathcal{L I P}}(\alpha \rightarrow \beta) \rightarrow((\gamma \bullet \alpha) \rightarrow(\gamma \bullet \beta)) \quad$ by 7,8, Ax W2

Proposition 7.3 Let $\alpha, \beta \in$ Term $_{\sqrt{q \mathcal{P M V}}}$ and $T \subseteq$ Term $_{\sqrt{q \mathcal{P M V}}}$. Then:

1. Axioms of $\mathcal{L} \mathcal{I P}$ are tautologies.

2. $\{\alpha, \alpha \rightarrow \beta\} \mid={ }_{\mathcal{I} P}^{\text {Prob }} \beta$.

3. If $T \vdash_{\mathcal{L I P}} \alpha$ then $T=_{\mathcal{I} \mathcal{P}}^{\text {rob }} \alpha$.

Proof: 1) Immediate. 2) Let $\alpha, \beta \in$ Term $_{\sqrt{q \mathcal{P M V}}}$ and $e: \sqrt{q \mathcal{P M \mathcal { V }}} \rightarrow A$ be an $\mathcal{I P}$-valuation such that $e_{p}(\alpha \rightarrow \beta)=1$ and $e_{p}(\alpha)=1$. We show that $e_{p}(\beta)=1$. In fact: $1=e_{p}(\alpha \rightarrow \beta)=e((\alpha \rightarrow \beta) \oplus 0)=e(\alpha \oplus 0) \rightarrow(\beta \oplus 0)$ and then $e_{p}(\alpha)=e(\alpha \oplus 0) \leq e(\beta \oplus 0)=e_{p}(\beta)$. Therefore $e_{p}(\alpha)=1$ implies that $1=e_{p}(\beta)$ since $1 \leq e(\beta \oplus 0)=e(\beta) \oplus 0 \in \operatorname{Reg}(A)$ and $\operatorname{Reg}(A)$ is a $P M V$-algebra (see Proposition 4.5-1). 3) Immediate from items 1 and 2.

Now we focus on a sub-calculus of $\mathcal{L} \mathcal{I} \mathcal{P}$ particularly relevant in the study of the completeness of $\mathcal{L I P}$. Consider the set $V \cup(\sqrt{x})_{x \in V}$ where $V$ is the usual set of variables in Term $\sqrt{q \mathcal{P M V}}$. Let $\operatorname{Term}_{\sqrt{V}}$ be the smallest set $S \subseteq$ Term $_{\sqrt{q \mathcal{P M V}}}$ such that $\left\{0, \frac{1}{2}, 1\right\} \cup V \cup(\sqrt{x})_{x \in V} \subseteq S$ and if $\alpha, \beta \in S$ then, $\neg \alpha \in S$ and $\alpha \star \beta \in S$ where $\star \in\{\oplus, \bullet\}$. Now we define the Hilbertstyle calculus

$$
\mathcal{L}_{\sqrt{V}}=\left\langle\operatorname{Term}_{\sqrt{V}}, \vdash_{\mathcal{L}_{\sqrt{V}}}\right\rangle
$$

given by the axioms $\mathrm{W} 1 \ldots \mathrm{W} 4, \mathrm{C} 1 \ldots \mathrm{C} 4, \mathrm{P} 1 \ldots \mathrm{P} 5$ and $\mathrm{MP}$ as inference rule.

Remark 7.4 By definition of $\mathcal{L}_{\sqrt{V}}$, the results of Lemma 7.2 continue to be valid in $\mathcal{L}_{\sqrt{V}}$.

Let $T \subseteq T e r m_{\sqrt{V}}$ i.e., a theory in $\operatorname{Term}_{\sqrt{V}}$. Then $T$ is said to be complete in $\mathcal{L}_{\sqrt{V}}$ iff for each pair of terms $\alpha, \beta$ in $\operatorname{Term}_{\sqrt{V}} ; T \vdash_{\sqrt{V}} \alpha \rightarrow \beta$ or $T \vdash_{\sqrt{V}} \beta \rightarrow \alpha$. Moreover $T$ is inconsistent in $\mathcal{L}_{\sqrt{V}}$ iff $T \vdash_{\sqrt{V}} \alpha$ for each $\alpha \in \operatorname{Term}_{\sqrt{V}}$, otherwise it is consistent in $\mathcal{L}_{\sqrt{V}}$.

Lemma 7.5 Let $T$ be a theory and $\alpha$ be a term, both in Term $\sqrt{V}$. Suppose that $T \forall_{\sqrt{V}} \alpha$. Then there exists a complete theory $T^{\prime}$ in $T e r m_{\sqrt{V}}$ such that, $T \subseteq T^{\prime}$ and $T^{\prime} \forall_{\sqrt{V}} \alpha$. 
Proof: It follows by the same arguments used in [13, Lemma 2.4.2].

Let $A$ be a $P M V_{\frac{1}{2^{4}}}$-algebra. Term $\sqrt{V}^{\text {-valuations }}$ in $A$ are functions $v: \operatorname{Term}_{\sqrt{V}} \rightarrow A$ satisfying $v(0)=0, v\left(\frac{1}{2}\right)=\frac{1}{2}, v(1)=1, v(\neg \alpha)=\neg v(\alpha)$ and $v(\alpha \star \beta)=v(\alpha) \star v(\beta)$ where $\star \in\{\oplus, \bullet\}$. Note that for a Term $\sqrt{V}^{-}$ valuation, the terms in the set $(\sqrt{x})_{x \in V}$ have no restriction in the election of the value $v(\sqrt{x})$.

Theorem 7.6 Let $T$ be a consistent theory in Term $_{\sqrt{V}}$. For each $\alpha \in$ Term $_{\sqrt{V}}$ we consider the class

$$
[\alpha]=\left\{\beta \in \operatorname{Term}_{\sqrt{V}}: T \vdash_{\mathcal{L}_{\sqrt{V}}} \alpha \leftrightarrow \beta\right\}
$$

Let $L_{T}=\left\{[\alpha]: \alpha \in\right.$ Term $\left._{\sqrt{V}}\right\}$. If we define the following operations in $L_{T}$ :

$$
\begin{array}{ll}
0=[0] & \neg[\alpha]=[\neg \alpha] \\
\frac{1}{2}=\left[\frac{1}{2}\right] & {[\alpha] *[\beta]=[\alpha * \beta] \text { for } * \in\{\oplus, \bullet\}} \\
1=[1] &
\end{array}
$$

Then:

1. $\left\langle L_{T}, \oplus, \bullet, \neg, 0, \frac{1}{2}, 1\right\rangle$ is a $P M V_{\frac{1}{2^{4}}}$-algebra.

2. If $\alpha \in T$ then $[\alpha]=1$.

3. If $T$ is a complete theory then $L_{T}$ is a totally ordered set.

Proof: 1) We first show that the operations are well defined on $L_{T}$. In the cases $\oplus, \neg, 0, \frac{1}{2}, 1$ we refer to [13, Lemma 2.3.12]. The case $\bullet$ follows from Lemma $7.2-11$. By axioms $\mathrm{W} 1 \ldots \mathrm{W} 4, \mathrm{C} 1 \ldots \mathrm{C} 4, \mathrm{P} 1 \ldots \mathrm{P} 5$, it is not very hard to see that $L_{T}$ is a $P M V_{\frac{1}{2^{4}}}$-algebra. 2) Follows from axiom W1. 3) Follows using the same argument as in [13, Lemma 2.4.2].

We will refer to $L_{T}$ as the Lindenbaum algebra associated to the theory $T \subseteq \operatorname{Term}_{\sqrt{V}}$. Clearly, the natural application $\alpha \mapsto[\alpha]$ is a Term $\sqrt{V}^{-}$ valuation in $L_{T}$.

Definition 7.7 We define the $\sqrt{V}$-translation $\alpha \stackrel{t}{\rightarrow} \alpha_{t}$ as the application $t: \operatorname{Term}_{\sqrt{q \mathcal{P M V}}} \rightarrow \operatorname{Term}_{\sqrt{V}}$ such that: 
$x \stackrel{t}{\mapsto} x$ and $\sqrt{x} \stackrel{t}{\mapsto} \sqrt{x} \quad$ for each $x \in V$,

$c \stackrel{t}{\mapsto} c$ and $\sqrt{c} \stackrel{t}{\mapsto} \frac{1}{2} \quad$ for each $c \in\left\{0, \frac{1}{2}, 1\right\}$,

$\neg \alpha \stackrel{t}{\mapsto} \neg\left(\alpha_{t}\right)$,

$\sqrt{\neg \alpha} \stackrel{t}{\mapsto}(\neg \sqrt{\alpha})_{t}$,

$\sqrt{\alpha \star \beta} \stackrel{t}{\mapsto} \frac{1}{2} \quad$ for each binary connective $\star$,

$\alpha \star \beta \stackrel{t}{\mapsto} \alpha_{t} \star \beta_{t} \quad$ for each binary connective $\star$

If $T$ is a theory in $\operatorname{Term}_{\sqrt{q \mathcal{P M V V}}}$, then we define the $\sqrt{V}$-translation over $T$ as the set $T_{t}=\left\{\alpha_{t}: \alpha \in T\right\}$.

Proposition 7.8 Let $\alpha \in$ Term $_{\sqrt{q \mathcal{P M V}}}$. Then:

$$
\vdash_{\mathcal{L I P}} \alpha \leftrightarrow \alpha_{t}
$$

Proof: We use induction on the complexity of terms. Let $\alpha$ be an atomic term. By definition of $\sqrt{V}$-translation, Lemma $7.2-1$ and axiom sQ3 of $\mathcal{L I P}$ it is clear that $\vdash_{\mathcal{L I P}} \alpha \leftrightarrow \alpha_{t}$ and $\vdash_{\mathcal{L I P}} \sqrt{\alpha} \leftrightarrow(\sqrt{\alpha})_{t}$.

Suppose that $\vdash_{\mathcal{L I P}} \alpha \leftrightarrow \alpha_{t}$ and $\vdash_{\mathcal{L I P}} \beta \leftrightarrow \beta_{t}$.

- By Lemma 7.2-6 we have that $\vdash_{\mathcal{L I P}} \neg \alpha \leftrightarrow \neg \alpha_{t}$.

- Let $\star \in\{\oplus, \bullet\}$. Then we have that:

(1) $\vdash_{\mathcal{L I P}} \alpha \rightarrow \alpha_{t}$

(2) $\vdash_{\mathcal{L I P}}\left(\alpha \rightarrow \alpha_{t}\right) \rightarrow\left((\alpha \star \beta) \rightarrow\left(\alpha_{t} \star \beta\right)\right) \quad$ by Lemma 7.2 , item 7 or 11

$(3) \vdash_{\mathcal{L I P}}(\alpha \star \beta) \rightarrow\left(\alpha_{t} \star \beta\right)$ MP 1-2

(4) $\vdash_{\mathcal{L I P}} \beta \rightarrow \beta_{t}$

(5) $\vdash_{\mathcal{L I P}}\left(\beta \rightarrow \beta_{t}\right) \rightarrow\left(\left(\alpha_{t} \star \beta\right) \rightarrow\left(\alpha_{t} \star \beta_{t}\right)\right)$ by Lemma 7.2 , item 7 or 11

(6) $\vdash_{\mathcal{L I P}}\left(\alpha_{t} \star \beta\right) \rightarrow\left(\alpha_{t} \star \beta_{t}\right)$

MP 4-5

(7) $\vdash_{\mathcal{L I P}}(\alpha \star \beta) \rightarrow\left(\alpha_{t} \star \beta_{t}\right)$

by Lemma $7.2-4$

By the same argument we can prove that $\vdash_{\mathcal{L I P}}\left(\alpha_{t} \star \beta_{t}\right) \rightarrow(\alpha \star \beta)$.

Hence $\vdash_{\mathcal{L I P}}(\alpha \star \beta) \leftrightarrow(\alpha \star \beta)_{t}$. 
- If $\alpha$ is $\sqrt{\gamma}$ then we have to consider two cases:

i) $\gamma$ is $\gamma_{1} \star \gamma_{2}$ such that $\star \in\{\oplus, \bullet\}$. Then $\alpha_{t}=(\sqrt{\gamma})_{t}=\left(\sqrt{\gamma_{1} \star \gamma_{2}}\right)_{t}=\frac{1}{2}$. By Axiom sQ2, $\vdash_{\mathcal{L I P}}(\sqrt{\gamma}) \leftrightarrow \frac{1}{2}$. Hence $\vdash_{\mathcal{L I P}} \alpha \leftrightarrow \alpha_{t}$.

ii) $\gamma$ is $\sqrt{\gamma_{1}}$. Then $\alpha_{t}=\left(\sqrt{\sqrt{\gamma_{1}}}\right)_{t}=\left(\neg \gamma_{1}\right)_{t}=\neg\left(\gamma_{1}\right)_{t}$. By inductive hypothesis $\vdash_{\mathcal{L I P}} \gamma_{1} \leftrightarrow\left(\gamma_{1}\right)_{t}$ and then $\vdash_{\mathcal{L I P}} \neg \gamma_{1} \leftrightarrow \neg\left(\gamma_{1}\right)_{t}$. Hence $\vdash_{\mathcal{L I P}} \sqrt{\sqrt{\gamma_{1}}} \leftrightarrow \neg \gamma_{1}$ and $\vdash_{\mathcal{L I P}} \alpha \leftrightarrow \alpha_{t}$.

Taking into account the axiom sQ4,... sQ7, we introduce, in the following definition, the theory $T_{D} \subseteq$ Term $_{\sqrt{V}}$ which will allow to establish a relation between proofs in $\mathcal{L I P}$ and proofs in $\mathcal{L}_{\sqrt{V}}$.

Definition 7.9 We consider the following sets of terms in $\operatorname{Term}_{\sqrt{V}}$

$$
\begin{aligned}
& T_{1}=\left\{\left(\frac{1}{2^{4}} \oplus\left(\frac{s \bullet s}{2^{2}} \oplus \frac{\sqrt{s} \bullet \sqrt{s}}{2^{2}}\right)\right) \rightarrow\left(\frac{s}{2^{2}} \oplus \frac{\sqrt{s}}{2^{2}}\right): s \in V \cup\left\{0, \frac{1}{2}, 1\right\}\right\}, \\
& T_{2}=\left\{\left(\frac{1}{2^{4}} \oplus\left(\frac{\neg s \bullet \neg s}{2^{2}} \oplus \frac{\sqrt{s} \bullet \sqrt{s}}{2^{2}}\right)\right) \rightarrow\left(\frac{\neg s}{2^{2}} \oplus \frac{\sqrt{s}}{2^{2}}\right): s \in V \cup\left\{0, \frac{1}{2}, 1\right\}\right\}, \\
& T_{3}=\left\{\left(\frac{1}{2^{4}} \oplus\left(\frac{s \bullet s}{2^{2}} \oplus \frac{\sqrt{\neg s} \bullet \sqrt{\neg s}}{2^{2}}\right)\right) \rightarrow\left(\frac{s}{2^{2}} \oplus \frac{\sqrt{\neg s}}{2^{2}}\right): s \in V \cup\left\{0, \frac{1}{2}, 1\right\}\right\}, \\
& T_{4}=\left\{\left(\frac{1}{2^{4}} \oplus\left(\frac{\neg s \bullet \neg s}{2^{2}} \oplus \frac{\sqrt{\neg s} \bullet \sqrt{\neg s}}{2^{2}}\right)\right) \rightarrow\left(\frac{\neg s}{2^{2}} \oplus \frac{\sqrt{\neg s}}{2^{2}}\right): s \in V \cup\left\{0, \frac{1}{2}, 1\right\} .\right.
\end{aligned}
$$

Then we define:

$$
T_{D}=T_{1} \cup T_{2} \cup T_{3} \cup T_{4}
$$

Proposition 7.10 Let $\alpha \in$ Term $_{\sqrt{q \mathcal{P M V}}}$. Then:

$$
\begin{aligned}
& \text { 1. } T_{D} \vdash_{\mathcal{L}_{\sqrt{V}}}\left(\left(\frac{1}{2^{4}} \oplus\left(\frac{\alpha \bullet \alpha}{2^{2}} \oplus \frac{\sqrt{\alpha} \bullet \sqrt{\alpha}}{2^{2}}\right)\right) \rightarrow\left(\frac{\alpha}{2^{2}} \oplus \frac{\sqrt{\alpha}}{2^{2}}\right)\right)_{t} . \\
& \text { 2. } T_{D} \vdash_{\mathcal{L}_{\sqrt{V}}}\left(\left(\frac{1}{2^{4}} \oplus\left(\frac{\neg \alpha \bullet \neg \alpha}{2^{2}} \oplus \frac{\sqrt{\alpha} \bullet \sqrt{\alpha}}{2^{2}}\right)\right) \rightarrow\left(\frac{\neg \alpha}{2^{2}} \oplus \frac{\sqrt{\alpha}}{2^{2}}\right)\right)_{t} . \\
& \text { 3. } T_{D} \vdash_{\mathcal{L}_{\sqrt{V}}}\left(\left(\frac{1}{2^{4}} \oplus\left(\frac{\alpha \bullet \alpha}{2^{2}} \oplus \frac{\sqrt{\neg \alpha} \bullet \sqrt{\neg \alpha}}{2^{2}}\right)\right) \rightarrow\left(\frac{\alpha}{2^{2}} \oplus \frac{\sqrt{\neg \alpha}}{2^{2}}\right)\right)_{t} . \\
& \text { 4. } T_{D} \vdash_{\mathcal{L}_{\sqrt{V}}}\left(\left(\frac{1}{2^{4}} \oplus\left(\frac{\neg \alpha \bullet \neg \alpha}{2^{2}} \oplus \frac{\sqrt{\neg \alpha} \bullet \sqrt{\neg \alpha}}{2^{2}}\right)\right) \rightarrow\left(\frac{\neg \alpha}{2^{2}} \oplus \frac{\sqrt{\neg \alpha}}{2^{2}}\right)\right)_{t} .
\end{aligned}
$$

Proof: $\quad$ Let $\alpha \in$ Term $_{\sqrt{q \mathcal{P M V}}}$. For the sake of simplicity we use the following notation:

$$
\alpha_{t}^{1} \text { means }\left(\left(\frac{1}{2^{4}} \oplus\left(\frac{\alpha \bullet \alpha}{2^{2}} \oplus \frac{\sqrt{\alpha} \bullet \sqrt{\alpha}}{2^{2}}\right)\right) \rightarrow\left(\frac{\alpha}{2^{2}} \oplus \frac{\sqrt{\alpha}}{2^{2}}\right)\right)_{t},
$$




$$
\begin{aligned}
& \alpha_{t}^{2} \text { means }\left(\left(\frac{1}{2^{4}} \oplus\left(\frac{\neg \alpha \bullet \neg \alpha}{2^{2}} \oplus \frac{\sqrt{\alpha} \bullet \sqrt{\alpha}}{2^{2}}\right)\right) \rightarrow\left(\frac{\neg \alpha}{2^{2}} \oplus \frac{\sqrt{\alpha}}{2^{2}}\right)\right)_{t}, \\
& \alpha_{t}^{3} \text { means }\left(\left(\frac{1}{2^{4}} \oplus\left(\frac{\alpha \cdot \alpha}{2^{2}} \oplus \frac{\sqrt{\neg \alpha} \cdot \sqrt{\neg \alpha}}{2^{2}}\right)\right) \rightarrow\left(\frac{\alpha}{2^{2}} \oplus \frac{\sqrt{\neg \alpha}}{2^{2}}\right)\right)_{t}, \\
& \alpha_{t}^{4} \text { means }\left(\left(\frac{1}{2^{4}} \oplus\left(\frac{\neg \alpha \bullet \neg \alpha}{2^{2}} \oplus \frac{\sqrt{\neg \alpha} \cdot \sqrt{\neg \alpha}}{2^{2}}\right)\right) \rightarrow\left(\frac{\neg \alpha}{2^{2}} \oplus \frac{\sqrt{\neg \alpha}}{2^{2}}\right)\right)_{t} .
\end{aligned}
$$

We use induction on the complexity of $\alpha$.

- The case $\alpha \in V \cup\left\{0, \frac{1}{2}, 1\right\}$ is immediate from definition of $T_{D}$.

- Suppose that $\alpha$ is $\alpha_{1} \star \alpha_{2}$ where $\star \in\{\oplus, \bullet\}$.

By Axiom P3, Axiom C4 and Lemma 7.2-4 it follows that for each $\alpha \in$ $\operatorname{Term}_{\sqrt{V}}$

$$
\vdash_{\mathcal{L}_{\sqrt{V}}}\left(\frac{1}{2^{4}} \oplus\left(\frac{\alpha \bullet \alpha}{2^{2}} \oplus \frac{1}{2^{4}}\right)\right) \rightarrow\left(\frac{\alpha}{2^{2}} \oplus \frac{1}{2^{3}}\right)
$$

We prove that $T_{D} \vdash_{\mathcal{L}_{\sqrt{V}}} \alpha_{t}^{1}$. By definition of $\sqrt{V}$-translation we have that $\alpha_{t}^{1}=\left(\left(\frac{1}{2^{4}} \oplus\left(\frac{\left(\alpha_{1} \star \alpha_{2}\right) \bullet\left(\alpha_{1} \star \alpha_{2}\right)}{2^{2}} \oplus \frac{\sqrt{\alpha_{1} \star \alpha_{2}} \bullet \sqrt{\alpha_{1} \star \alpha_{2}}}{2^{2}}\right)\right) \rightarrow\left(\frac{\alpha_{1} \star \alpha_{2}}{2^{2}} \oplus \frac{\sqrt{\alpha_{1} \star \alpha_{2}}}{2^{2}}\right)\right)_{t}=$ $\left(\frac{1}{2^{4}} \oplus\left(\frac{\left(\alpha_{1} \star \alpha_{2}\right)_{t} \bullet\left(\alpha_{1} \star \alpha_{2}\right)_{t}}{2^{2}} \oplus \frac{1}{2^{4}}\right) \rightarrow\left(\left(\frac{\left.\alpha_{1} \star \alpha_{2}\right)_{t}}{2^{2}} \oplus \frac{1}{2^{3}}\right)\right.\right.$. Since $\left(\alpha_{1} \star \alpha_{2}\right)_{t} \in \operatorname{Term}_{\sqrt{V}}$, by (1), we have $\vdash_{\mathcal{L}_{\sqrt{V}}} \alpha_{t}^{1}$ and $T_{D} \vdash_{\mathcal{L}_{\sqrt{V}}} \alpha_{t}^{1}$. Cases $T_{D} \vdash_{\mathcal{L}_{\sqrt{V}}} \alpha_{t}^{2}, T_{D} \vdash_{\mathcal{L}_{\sqrt{V}}} \alpha_{t}^{3}$ and $T_{D} \vdash_{\mathcal{L}_{\sqrt{V}}} \alpha_{t}^{4}$ follow in a similar way.

- Suppose $\alpha$ is $\sqrt{\beta}$.

We prove that $T_{D} \vdash_{\mathcal{L}_{\sqrt{V}}} \alpha_{t}^{1}$. By definition of $\sqrt{V}$-translation we have that $\alpha_{t}^{1}=\left(\left(\frac{1}{2^{4}} \oplus\left(\frac{\sqrt{\beta} \bullet \sqrt{\beta}}{2^{2}} \oplus \frac{\sqrt{\sqrt{\beta} \bullet \sqrt{\sqrt{\beta}}}}{2^{2}}\right)\right) \rightarrow\left(\frac{\sqrt{\beta}}{2^{2}} \oplus \frac{\sqrt{\sqrt{\beta}}}{2^{2}}\right)\right)_{t}=\left(\left(\frac{1}{2^{4}} \oplus\left(\frac{(\sqrt{\beta})_{t} \bullet(\sqrt{\beta})_{t}}{2^{2}} \oplus\right.\right.\right.$ $\left.\left.\frac{\neg \beta_{t} \bullet \neg \beta_{t}}{2^{2}}\right)\right) \rightarrow\left(\frac{(\sqrt{\beta})_{t}}{2^{2}} \oplus \frac{\neg \beta_{t}}{2^{2}}\right)$. By inductive hypothesis we have $T_{D} \vdash_{\mathcal{L}_{\sqrt{V}}} \beta_{t}^{2}$ and by Lemma 7.2 it is straightforward to see that

$$
T_{D} \vdash_{\mathcal{L}_{\sqrt{V}}} \beta_{t}^{2} \leftrightarrow\left(\left(\frac{1}{2^{4}} \oplus\left(\frac{(\sqrt{\beta})_{t} \bullet(\sqrt{\beta})_{t}}{2^{2}} \oplus \frac{\neg \beta_{t} \bullet \neg \beta_{t}}{2^{2}}\right)\right) \rightarrow\left(\frac{(\sqrt{\beta})_{t}}{2^{2}} \oplus \frac{\neg \beta_{t}}{2^{2}}\right)\right)
$$

Hence, by Lemma 7.2-3, $T_{D} \vdash_{\mathcal{L}_{\sqrt{V}}} \alpha_{t}^{1}$. Cases $T_{D} \vdash_{\mathcal{L}_{\sqrt{V}}} \alpha_{t}^{2}, T_{D} \vdash_{\mathcal{L}_{\sqrt{V}}} \alpha_{t}^{3}$ and $T_{D} \vdash_{\mathcal{L}_{\sqrt{V}}} \alpha_{t}^{4}$ follow in a similar way.

Theorem 7.11 Let $\alpha \in T e r m_{\sqrt{q \mathcal{P M V}}}$ and $T \subseteq T e r m_{\sqrt{q \mathcal{P} \mathcal{M V}}}$. Then:

$$
T \vdash_{\mathcal{L I P}} \alpha \quad \text { iff } \quad T_{t} \cup T_{D} \vdash_{\mathcal{L}_{\sqrt{V}}} \alpha_{t}
$$


Proof: Suppose that $T \vdash_{\mathcal{L I P}} \alpha$. We use induction on the length of the proof of $\alpha$ noted by Length $(\alpha)$. If Length $(\alpha)=1$ then we have the following possibilities:

1. $\alpha$ is one of the axioms $\mathrm{W} 1 \ldots \mathrm{W} 4, \mathrm{C} 1 \ldots \mathrm{C} 4, \mathrm{P} 1 \ldots \mathrm{P} 5$. In this case $\alpha_{t}$ results an axiom of $\mathcal{L}_{\sqrt{V}}$ and $\vdash_{\mathcal{L}_{\sqrt{V}}} \alpha_{t}$.

2. $\alpha$ is one of the axioms sQ1...sQ3. In this case $\alpha_{t}$ looks like $\beta \leftrightarrow \beta$ in $\operatorname{Term}_{\sqrt{V}}$. Then, by Proposition 7.2-1, $\vdash_{\mathcal{L}_{\sqrt{V}}} \alpha_{t}$.

3. $\alpha$ is one of the axioms SQ4...sQ7. In this case, by Proposition 7.10, $T_{D} \vdash_{\mathcal{L}_{\sqrt{V}}} \alpha_{t}$.

4. If $\alpha \in T$ then $\alpha_{t} \in T_{t}$. Hence, $T_{t} \vdash_{\mathcal{L}_{\sqrt{V}}} \alpha_{t}$.

Suppose that the theorem is valid for $\operatorname{Length}(\alpha)<n$. We consider $\operatorname{Lengh}(\alpha)=n$. Thus we have a proof of $\alpha$ from $T$ as follows

$$
\alpha_{1}, \cdots, \alpha_{m} \rightarrow \alpha, \cdots, \alpha_{m}, \cdots, \alpha_{n-1}, \alpha
$$

obtaining $\alpha$ by MP from $\alpha_{m} \rightarrow \alpha$ and $\alpha_{m}$. Using the inductive hypothesis we have that $T_{t} \cup T_{D} \vdash_{\mathcal{L}_{\sqrt{V}}}\left(\alpha_{m} \rightarrow \alpha\right)_{t}$ and $T_{t} \cup T_{D} \vdash_{\mathcal{L}_{\sqrt{V}}}\left(\alpha_{m}\right)_{t}$. Taking into account that $\left(\alpha_{m} \rightarrow \alpha\right)_{t}=\left(\alpha_{m}\right)_{t} \rightarrow \alpha_{t}$, by MP, we have $T_{t} \cup T_{D} \vdash_{\mathcal{L}_{\sqrt{V}}} \alpha_{t}$.

For the converse, suppose that $T_{t} \cup T_{D} \vdash_{\mathcal{L}_{\sqrt{V}}} \alpha_{t}$. Then there exist two subsets $\left\{\beta_{1}, \cdots, \beta_{n}\right\} \subseteq T$ and $\left\{\gamma_{1}, \cdots, \gamma_{m}\right\} \subseteq T_{D}$ such that

$$
\left\{\left(\beta_{1}\right)_{t}, \cdots,\left(\beta_{n}\right)_{t}, \gamma_{1}, \cdots, \gamma_{m}\right\} \vdash_{\mathcal{L}_{\sqrt{V}}} \alpha_{t}
$$

Consequently $\left\{\left(\beta_{1}\right)_{t}, \cdots,\left(\beta_{n}\right)_{t}, \gamma_{1}, \cdots, \gamma_{m}\right\} \vdash_{\mathcal{L I P}} \alpha_{t}$. By Lemma 7.8 we have that $\vdash_{\mathcal{L I P}} \alpha \leftrightarrow \alpha_{t}$ and $\vdash_{\mathcal{L I P}} \beta_{i} \leftrightarrow\left(\beta_{i}\right)_{t}$ for each $i \in\{1, \cdots, n\}$. Moreover, by Axiom sQ4...sQ7, $\vdash_{\mathcal{L I P}} \gamma_{j}$ for each $j \in\{1, \cdots, m\}$. Thus $\left\{\beta_{1}, \cdots, \beta_{n}\right\} \vdash_{\mathcal{L I P}}$ $\alpha$ and $T \vdash_{\mathcal{L I P}} \alpha$.

Corollary 7.12 Let $\alpha \in$ Term $\sqrt{q \mathcal{P M N}}$. Then, $\vdash_{\mathcal{L I P}} \alpha$ iff $T_{D} \vdash_{\mathcal{L}_{\sqrt{V}}} \alpha_{t}$.

Let $S_{A}$ be the pair algebra over the $\mathcal{P} \mathcal{M V}{ }_{\frac{1}{24}}$-chain $A$. Consider the sub-algebra $D_{A}$ of $S_{A}$ (i.e. the $I P$-algebra defined in Proposition 5.5). We introduce the following sets:

$$
E_{D_{A}}=\left\{\text { valuations } e: \operatorname{Term}_{\sqrt{q \mathcal{P M \mathcal { V }}}} \rightarrow D_{A}\right\}
$$




$$
V_{D_{A}}=\left\{\operatorname{Term} \sqrt{V}^{\text {-valuations }} v: \operatorname{Term} m_{\sqrt{V}} \rightarrow \operatorname{Reg}\left(D_{A}\right) \text { s.t. } v\left(T_{D}\right)=1\right\}
$$

Proposition 7.13 Let $e \in E_{D_{A}}$ and the restriction $v_{e}=\left.e_{p}\right|_{\text {Term }}{ }_{\sqrt{q \mathcal{P M V}}}$ where $e_{p}(t)$ is the generalized probability value. Then the assignment $e \mapsto v_{e}$ is a bijection $E_{D_{A}} \rightarrow V_{D}$ such that $e_{p}(\alpha)=v_{e}\left(\alpha_{t}\right)$.

Proof: We first prove that $e \mapsto v_{e}$ is well defined in the sense that $v_{e} \in V_{D}$. Let $\alpha \in T_{D}$. Then $v_{e}(\alpha)=e_{p}(\alpha)=e(\alpha \oplus 0)=e(\alpha)=1$ since $D_{A} \in \mathcal{I P}$. Hence $v_{e}\left(T_{D}\right)=1$.

We prove the injectivity. Suppose that $v_{e_{1}}=v_{e_{2}}$. Let $t$ be an atomic term in $\operatorname{term}_{\mathcal{I} \mathcal{P}}$. Then we have that $e_{1 p}(t)=v_{e_{1}}(t)=v_{e_{2}}(t)=e_{2 p}(t)$ and $e_{1 p}(\sqrt{t})=v_{e_{1}}(\sqrt{t})=v_{e_{2}}(\sqrt{t})=e_{2 p}(\sqrt{t})$. Therefore by Proposition 6.5, $e_{1}=e_{2}$ and $e \mapsto v_{e}$ is injective.

Now we prove the surjectivity. Let $v \in V_{D_{A}}$. By Proposition 5.4, consider the $\mathcal{P} \mathcal{M} \mathcal{V}_{\frac{1}{2^{4}}}$-isomorphism $g: \operatorname{Reg}\left(D_{A}\right) \rightarrow A$ given by $g\left(a, \frac{1}{2}\right)=a$ and define the valuation $e: \operatorname{Term}_{\sqrt{q \mathcal{P M} \mathcal{V}}} \rightarrow D_{A}$ such that for each atomic term $t$ in $\operatorname{Term}_{\sqrt{q \mathcal{P \mathcal { M }}}} e(t)=(g v(t), g v(\sqrt{t}))$. By induction on the complexity of terms we prove that $v_{e}=v$.

Let $t$ be an atomic term in $\operatorname{Term}_{\sqrt{V}}$.

- The case $t \in\left\{0, \frac{1}{2}, 1\right\}$ is immediate.

- If $t$ is a variable $x$ then, $v_{e}(x)=e_{p}(x)=e(x \oplus 0)=e(x) \oplus 0=$ $(g v(x), g v(\sqrt{x})) \oplus 0=\left(g v(x), \frac{1}{2}\right)=v(x)$.

- If $t$ is $\sqrt{x}$ where $x$ is variable then, $v_{e}(\sqrt{x})=e_{p}(\sqrt{x})=e(\sqrt{x} \oplus 0)=$ $e(\sqrt{x}) \oplus 0=\sqrt{e(x)} \oplus 0=\sqrt{(g v(x), g v(\sqrt{x}))} \oplus 0=(g v(\sqrt{x}), \neg g v(x)) \oplus$ $0=\left(g v(\sqrt{x}), \frac{1}{2}\right)=v(\sqrt{x})$.

That constitutes the base of the induction in the language Term $\sqrt{V}$.

Now let our claim hold whenever the complexity of $\operatorname{Term}_{\sqrt{V}}$-terms is less than $n$ and $\alpha$ has complexity $n$.

- if $\alpha \in$ Term $_{\sqrt{V}}$ is $\alpha_{1} \star \alpha_{2}$ where $\star \in\{\oplus, \bullet\}$ then $e_{p}(\alpha)=e(\alpha \oplus 0)=$ $e\left(\left(\alpha_{1} \star \alpha_{2}\right) \oplus 0\right)=e\left(\left(\alpha_{1} \oplus 0\right) \star\left(\alpha_{2} \oplus 0\right)\right)=e\left(\alpha_{1} \oplus 0\right) \star e\left(\alpha_{2} \oplus 0\right)=$ $e_{p}\left(\alpha_{1}\right) \star e_{p}\left(\alpha_{2}\right)=v\left(\alpha_{1}\right) \star v\left(\alpha_{2}\right)=v\left(\alpha_{1} \star \alpha_{2}\right)$. 
- if $\alpha \in$ Term $_{\sqrt{V}}$ is $\neg \alpha_{1}$ then, $v_{e}(\alpha)=e_{p}\left(\neg \alpha_{1}\right)=e\left(\neg \alpha_{1} \oplus 0\right)=e\left(\neg\left(\alpha_{1} \oplus\right.\right.$ $0))=\neg e\left(\alpha_{1} \oplus 0\right)=\neg e_{p}\left(\alpha_{1}\right)=\neg v\left(\alpha_{1}\right)=v\left(\neg \alpha_{1}\right)=v(\alpha)$.

Thus $v=v_{e}$ and $e \mapsto v_{e}$ is a bijection from $E_{D_{A}}$ onto $V_{D_{A}}$.

Let $e \in E_{D_{A}}$. By induction on the complexity of terms we prove that for each $\alpha \in \operatorname{Term}_{\sqrt{q \mathcal{P} \mathcal{M V}}}, e_{p}(\alpha)=v_{e}\left(\alpha_{t}\right)$.

If $\alpha$ is an atomic term then $e_{p}(\alpha)=e_{p}\left(\alpha_{t}\right)=v_{e}\left(\alpha_{t}\right)$. Now let our claim hold whenever the complexity of the term is less than $n$ and $\alpha$ have complexity $n$.

Suppose that $\alpha$ is $\alpha_{1} \star \alpha_{2}$ where $\star \in\{\oplus, \bullet\}$. Then $e_{p}(\alpha)=e_{p}\left(\alpha_{1} \star \alpha_{2}\right)=$ $e\left(\left(\alpha_{1} \star \alpha_{2}\right) \oplus 0\right)=e\left(\left(\alpha_{1} \oplus 0\right) \star\left(\alpha_{2} \oplus 0\right)\right)=e\left(\alpha_{1} \oplus 0\right) \star e\left(\alpha_{2} \oplus 0\right)=e_{p}\left(\alpha_{1}\right) \star e_{p}\left(\alpha_{2}\right)=$ $v_{e}\left(\alpha_{1 t}\right) \star v_{e}\left(\alpha_{2 t}\right)=v_{e}\left(\alpha_{1 t} \star \alpha_{2 t}\right)=v_{e}\left(\alpha_{t}\right)$.

Suppose that $\alpha$ is $\sqrt{\alpha_{1}}$. Let us consider the following cases:

- $\alpha_{1}$ is an atomic term. Then its follows from the fact that $\left(\sqrt{\alpha_{1}}\right)_{t}=$ $\sqrt{\alpha_{1}}$.

- $\alpha$ is $\sqrt{\sqrt{\alpha_{1}}}$. Then $e_{p}(\alpha)=e_{p}\left(\sqrt{\sqrt{\alpha_{1}}}\right)=\neg e_{p}\left(\alpha_{1}\right)=\neg v_{e}\left(\alpha_{1 t}\right)=$ $v_{e}\left(\neg \alpha_{1 t}\right)=v_{e}\left(\left(\sqrt{\sqrt{\alpha_{1}}}\right) t\right)=v_{e}(\alpha)$.

- $\alpha_{1}$ is $\sqrt{\alpha_{2} \star \alpha_{3}}$ where $\star \in\{\oplus, \bullet\}$. Then $e_{p}(\alpha)=e_{p}\left(\sqrt{\alpha_{2} \star \alpha_{3}}\right)=$ $\left(\frac{1}{2}, \frac{1}{2}\right)=e_{p}\left(\frac{1}{2}\right)=v_{e}\left(\left(\sqrt{\alpha_{2} \star \alpha_{3}}\right)_{t}\right)=v_{e}\left(\alpha_{t}\right)$.

Hence $e_{p}(\alpha)=v_{e}\left(\alpha_{t}\right)$ for each $\alpha \in \operatorname{Term}_{\sqrt{q \mathcal{P} \mathcal{M V}}}$.

Theorem 7.14 Let $T$ be a theory and $\alpha$ be a term both in Term $\sqrt{q \mathcal{P M N}}$ then

$$
T \models_{\mathcal{I P}}^{\text {Prob }} \alpha \quad \text { iff } \quad T \vdash_{\mathcal{L I P}} \alpha
$$

Proof: We assume that $T$ is consistent. Suppose that $T \models_{\mathcal{I} \mathcal{P}}^{\text {Prob }} \alpha$ but $T \forall_{\mathcal{L I P}} \alpha$. Then, by Theorem 7.11, $T_{t} \cup T_{D} \forall_{\mathcal{L}_{\sqrt{V}}} \alpha_{t}$. By Lemma 7.5 and Theorem 7.6, there exists a complete theory $T^{\prime} \subseteq$ Term $_{\sqrt{V}}$ such that $T_{t} \cup T_{D} \subseteq T^{\prime}, T^{\prime} \forall \mathcal{L}_{\sqrt{V}} \alpha_{t}, L_{T^{\prime}}$ is a totally ordered $P M V_{\frac{1}{2^{4}}}$-algebra. Thus

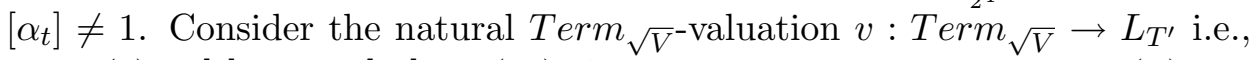
$s \mapsto v(s)=[s]$. Then $\left[\alpha_{t}\right]=v\left(\alpha_{t}\right) \neq 1$. Moreover, by Theorem 7.6, $v(\beta)=1$ for each $\beta \in T^{\prime}$.

By Proposition 7.13 there exits a valuation $e:$ Term $_{\sqrt{q \mathcal{P M V}}} \rightarrow D_{L_{T^{\prime}}}$ such that $e_{p}(\beta)=v\left(\beta_{t}\right)$ for each $\beta \in \operatorname{Term}_{\sqrt{q \mathcal{P M V}}}$. One the one hand, for 
each $\gamma \in T, e_{p}(\gamma)=v\left(\gamma_{t}\right)=1$ since $\gamma_{t} \in T^{\prime}$. Hence $e_{p}(T)=1$. On the other hand, $e_{p}(\alpha)=v\left(\alpha_{t}\right) \neq 1$ which is a contradiction since $T \models_{\mathcal{I P}}^{\text {Prob }} \alpha$. Thus $T \vdash_{\mathcal{L I P}} \alpha$. For the converse see Proposition 7.3.

Now we can establish a compactness theorem for the probabilistic consequence:

Theorem 7.15 Let $T$ be a theory and $\alpha$ be a term both in Term $m_{\mathcal{I P}}$. Then:

$$
T \models_{\mathcal{I} \mathcal{P}}^{\text {Prob }} \alpha \quad \text { iff } \quad \exists T_{0} \subseteq T \text { finite such that } T_{0} \models_{\mathcal{I} \mathcal{P}}^{\text {Prob }} \alpha
$$

Proof: $\quad$ If $T=_{\mathcal{I} P}^{\text {Prob }} \alpha$ by Theorem 7.14 there exists a proof of $\alpha, \alpha_{1}, \cdots \alpha_{n}, \alpha$ from $T$. If we consider $T_{0}=\left\{\alpha_{k} \in T: \alpha_{k} \in\left\{\alpha_{1}, \cdots \alpha_{n}\right\}\right\}$ then $T_{0} \models_{\mathcal{I} \mathcal{P}}^{P r o b} \alpha$. The converse is immediate.

\section{Conclusion}

In this paper we have developed a logical-algebraic study for the system of quantum computational gates known as Poincaré irreversible quantum computational system or $\mathbb{I P}$-system for short. The $\mathbb{I P}$-system is interesting not only due to its relation with the continuous $t$-norms but also because it may be possibly applicable to the study of error-correcting codes [20] in the context of quantum computation. Several algebraic structures originated in reducts of the $\mathbb{I P}$-system, as $q M V$-algebras and $\sqrt{q M V}$-algebras, were introduced and studied in recent years, remaining as an open problem that posed in [3] and [5] about the axiomatizability of the $\mathbb{I} \mathbb{P}$-system.

Facing this situation, the main results of this paper are the follwing: i) We have introduced an algebraic structure, the $I P$-algebra, that allows to give a mathematical representation of circuits made from assemblies of quantum gates of the $\mathbb{I P}$-system. ii) We have established a Hilbert-style calculus and a completeness theorem respect to the variety of $I P$-algebras, thus providing an answer to the mentioned open problem.

\section{References}

[1] D. Aharanov, A. Kitaev and N. Nisan: Quantum circuits with mixed states, Proc. 13th Annual ACM Symp. on Theory of Computation, 2030 (STOC,1997). 
[2] S. Burris and H.P. Sankappanavar: "A course in Universal Algebra", Graduate Text in Mathematics, Springer-Verlag, New York, (1981).

[3] G. Cattaneo, M. Dalla Chiara, R. Giuntini and R. Leporini: An unsharp logic from quantum computation, Int. J. Theor. Phys. 43, 1803-1817 (2001).

[4] R. Cignoli, M. I. D'Ottaviano and D. Mundici: "Algebraic foundations of many-valued reasoning, Kluwer, Dordrecht-Boston-London (2000).

[5] M. L. Dalla Chiara, R. Giuntini and R. Greechie: "Reasoning in Quantum Theory, Kluwer, Dordrecht (2004).

[6] M. L. Dalla Chiara, R. Giuntini and R. Leporini: Quantum computational logics. A Survey, In Trends in Logic: 50 Years of Studia Logica, V. F. Hendricks, J. Malinowski, (Editors), Kluwer Academic Publishers, Dordrecht, 213255 (2003).

[7] G. Domenech and H. Freytes: Fuzzy propositional logic associated with quantum computational gates, Int. J. Theor. Phys. 34, 228-261 (2006).

[8] H. Freytes, G. Sergioli and A. Aricó: Representing continuous t-norms in quantum computation with mixed states, J. Phys. A: Math. Theor. 43 (2010).

[9] R. Giuntini, A. Ledda and F. Paoli: Expanding quasi-MV algebras by a quantum operator, Studia Logica 87, 99-128 (2007).

[10] S. Gudder: Quantum computational logic, Int. J. Theor. Phys. 42, 39-47 (2003).

[11] S. Gudder and R. Greechie: Sequential products on effect algebras, Rep. Math. Phys. 49, 87-111 (2002).

[12] S. Gudder and R. Greechie: Uniqueness and order in sequential effect algebras, Int. J. Theor. Phys. 44, 755-770 (2005).

[13] P. Hájek: "Metamathematics of fuzzy logic", Kluwer, DordrechtBoston-London (1998).

[14] R. Horčik, P. Cintula, Product Lukasiewicz Logic, Archive of Math. Logic, 43, 477-503 (2004), 
[15] U. Höhle: Commutative, residuated l-monoids, In: Non-classical Logics and their applications to Fuzzy Subset, a Handbook on the Mathematical Foundations of Fuzzy Set Theory, U. Höhle, E. P. Klement, (Editors). Kluwer, Dordrecht, (1995).

[16] J.R. Isbell: Notes on ordered rings. Algebra Universalis 1, 393-399 (1971).

[17] E. P. Klement, R. Mesiar and E. Pap: "Triangular Norms", Kluwer, Dordrecht-Boston-London (2000).

[18] K. Kraus: "States, effects and operations", Springer-Verlag, Berlin, (1983).

[19] V. Kreinovich and L. Longpré: Fast quantum computation algorithms for handling probabilistic and interval uncertainty, Math. Log. Quart. 50, 507-518 (2004).

[20] E. L. Lawler and I.S. Sarkissian An algorithm for Ulams game and its application to error correcting codes, Inf. Process. Lett. 568993 (1995).

[21] A. Ledda, M. Konig, F. Paoli and R. Giuntini: $M V$ algebras and quantum computation, Studia Logica 82, 245-270 (2006).

[22] F. Montagna: An Algebraic Approach to Propositional Fuzzy logic, J. of Logic, Language and Information 9, 91-124 (2000).

[23] F. Montagna: Functorial Representation Theorems for $M V_{\delta}$ Algebras with Additional Operators, J. of Algebra 238, 99-125 (2001).

[24] D. Mundici and B. Riecăn: Probability on MV-algebras, In: Handbook of Measure Theory (E. Pap Ed.), 869-909, North Holland, Amsterdam, (2002).

[25] M.A. Nielsen and I.L. Chuang: "Quantum Computation and Quantum Information”, Cambridge University Press, Cambridge, (2000).

[26] F. Paoli, A. Ledda, R. Giuntini and H. Freytes: On some properties of $q M V$-algebras and $\sqrt{{ }^{1}} q M V$-algebras, Rep. Math. Logic, 44, 53-85 (2008).

[27] V. Tarasov: Quantum computer with Mixed States and Four-Valued Logic, J. Phys. A 35, 5207-5235 (2002). 
Hector Freytes e-mail: hfreytes@gmail.com hfreytes@dm.uba.ar

Graciela Domenech e-mail: domenech@iafe.uba.ar 